\title{
AN ALGORITHM FOR THE GRADE-TWO RHEOLOGICAL MODEL
}

\author{
SARA POLlOCK ${ }^{1, *}$ (1) AND L. Ridgway SCOTT $^{2}$
}

\begin{abstract}
We develop an algorithm for solving the general grade-two model of non-Newtonian fluids which for the first time includes inflow boundary conditions. The algorithm also allows for both of the rheological parameters to be chosen independently. The proposed algorithm couples a Stokes equation for the velocity with a transport equation for an auxiliary vector-valued function. We prove that this model is well posed using the algorithm that we show converges geometrically in suitable Sobolev spaces for sufficiently small data. We demonstrate computationally that this algorithm can be successfully discretized and that it can converge to solutions for the model parameters of order one. We include in the appendix a description of appropriate boundary conditions for the auxiliary variable in standard geometries.
\end{abstract}

Mathematics Subject Classification. 76A05, 35A15.

Received June 15, 2021. Accepted February 24, 2022.

\section{INTRODUCTION}

Non-Newtonian fluids are found in all aspects of life, from bodily fluids [28] to engine oil [12,27]. Rheology (non-Newtonian behavior) plays a significant role in manufacturing, including food $[17,19]$. Thus advances in modeling and simulation of non-Newtonian fluids can have broad impact.

Models of non-Newtonian fluids have been studied extensively for many years, but only recently have there been mathematical advances [10] that allow models for them to be understood more completely. This understanding now allows development of numerical solution methods with a new level of reliability. The grade-two model is the simplest of a family of models proposed by Rivlin and Ericksen [11,15] in which the stress-strain relationship involves derivatives of the fluid velocity. It has been widely studied, but to date no general numerical method has been proposed and analyzed for solving it.

There have been many different approaches to the grade-two model. In two dimensions, certain simplifications can be made if one of the parameters is eliminated, and this allows both rigorous analysis of the system in Lipschitz domains [15] and also extensive numerical analysis of effective discretization schemes [14]. However, in [15], it was assumed that the flow velocity was tangential to the boundary. Still in two dimensions, the paper [8] removed that restriction by imposing third-order boundary conditions on the inflow velocity.

However, different approaches were required for general parameters and in three dimensions [1,3-5]. Although the method proposed in [1] is quite general, it was developed and analyzed only in the case of tangential flow

Keywords and phrases. Non-Newtonian flow, grade-two fluid flow, inflow boundary conditions, convergence analysis.

1 Department of Mathematics, University of Florida, Gainesville, FL 32611, USA.

2 University of Chicago, Chicago, IL 60637, USA.

*Corresponding author: s.pollock@ufl.edu

(C) The authors. Published by EDP Sciences, SMAI 2022

This is an Open Access article distributed under the terms of the Creative Commons Attribution License (https://creativecommons.org/licenses/by/4.0), which permits unrestricted use, distribution, and reproduction in any medium, provided the original work is properly cited. 
(a)
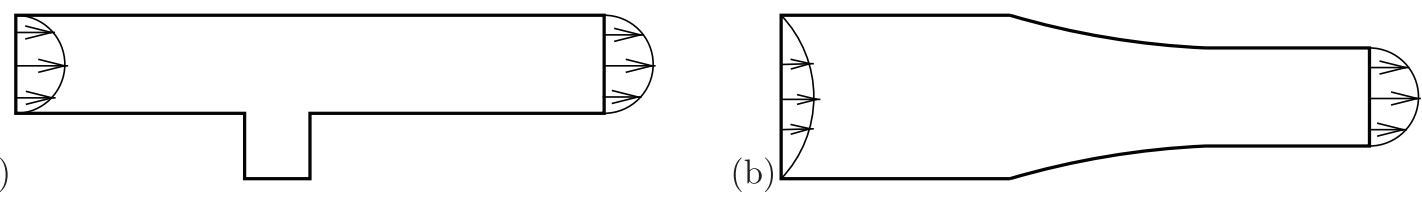

FiguRE 1. Two different rheometers: (a) the Lodge Stressmeter and (b) a contraction rheometer. The arrows on the left of each rheometer indicate the fluid inflow, and the arrows on the right of each rheometer indicate the fluid outflow.

fields. We propose here a slight variation of the approach [1] to the grade-two model that provides some simplifications both theoretically and computationally, and it applies in full generality.

For certain flow problems, it is critical to allow nontrivial inflow and outflow boundary conditions. For example, simulating many rheometers [21], the basic instruments for measuring fluid properties, requires this. In Figure 1 we depict two different rheometers which involve nontrivial inflow and outflow. The Lodge Stressmeter, depicted in Figure 4.13 of [2], was developed by Arthur Lodge [20]. The rheometer measurements are based on the pressure difference between the top of the middle section of the channel and the bottom of "hole" along the bottom of the channel. Contraction rheometers [23] measure the force on the contraction section in the middle between the large inflow channel and the smaller outflow channel. The shape of the contraction section can be chosen differently. Thus a major contribution of this paper is the development of analytical techniques to cover this type of boundary condition.

One issue with the different methods is the requirement for boundary conditions on the inflow boundary. Since the grade-two model is a third-order partial differential equation, we expect there to be another boundary condition in addition to the standard ones for flow problems, such as the Stokes no-slip condition. In [1], this issue was avoided by assuming tangential flow on the boundary. Generalizing [1] to allow an inflow boundary requires a boundary condition on a certain tensor $\Sigma$. In the approach proposed here, a condition is posed instead on the vector $-\Delta \mathbf{u}+\nabla p$, which is directly related to the divergence of the stress. Thus the additional boundary condition can be viewed as a stress boundary condition. We give examples of what this boundary condition should be for certain geometries.

\section{RHEOLOGY MODELS}

In all (time-independent) models of fluids, the basic equation can be written as

$$
\mathbf{u} \cdot \nabla \mathbf{u}+\nabla p=\nabla \cdot \mathbf{T}+\mathbf{f},
$$

where $\mathbf{T}$ is called the extra (also called deviatoric) stress and $\mathbf{f}$ represents externally given data. The models only differ according to the dependence of the stress on the velocity $\mathbf{u}$. In the case of a Newtonian fluid

$$
\mathbf{T}=\nu \mathbf{A},
$$

where $\mathbf{A}=\nabla \mathbf{u}+(\nabla \mathbf{u})^{t}$. Thus, when $\nabla \cdot \mathbf{u}=0$, it follows that $\nabla \cdot \mathbf{T}=\nu \Delta \mathbf{u}$, and we obtain the well known Navier-Stokes equations for Newtonian flow,

$$
-\nu \Delta \mathbf{u}+\mathbf{u} \cdot \nabla \mathbf{u}+\nabla p=\mathbf{f}
$$

where $\nu$ is the kinematic viscosity [18].

Typically, the data $\mathbf{f}$ is zero, but instead nonhomogeneous boundary conditions are physically relevant. Thus we will assume that (2.1) holds in some domain $\Omega$ and that $\mathbf{u}=\mathbf{g}$ on $\partial \Omega$, where we assume

$$
\int_{\partial \Omega} \mathbf{g} \cdot \mathbf{n} \mathrm{d} s=0
$$


to allow divergence-free solutions. Depending on the details of the model, there will also be a need for appropriate boundary conditions for $\mathbf{T}$ and other ingredients.

\subsection{Grade-two fluid model}

The grade-two model of Rivlin and Ericksen $[11,15]$ can be expressed as a single equation. The stress tensor for the grade-two fluid model satisfies

$$
\mathbf{T}_{\mathrm{G}}=\nu \mathbf{A}+\alpha_{1} \frac{\Delta}{\Delta t} \mathbf{A}+\alpha_{2} \mathbf{A}^{2}
$$

where $\mathbf{A}=(\nabla \mathbf{u})+(\nabla \mathbf{u})^{t}=2 \mathbf{E}$ and the material derivative and the lower-convected Oldroydian derivative are given by

$$
\frac{\mathrm{D}}{\mathrm{D} t} \mathbf{f}:=\left(\frac{\partial}{\partial t}+\mathbf{u} \cdot \nabla\right) \mathbf{f}, \quad \frac{\Delta}{\Delta t} \mathbf{f}:=\frac{\mathrm{D}}{\mathrm{D} t} \mathbf{f}+\mathbf{f}(\nabla \mathbf{u})+(\nabla \mathbf{u})^{t} \mathbf{f},
$$

for any tensor-valued function $\mathbf{f}$. For the steady-state, grade-two fluid model, the stress tensor simplifies to

$$
\mathbf{T}_{\mathrm{G}}=\nu \mathbf{A}+\alpha_{1}\left(\mathbf{u} \cdot \nabla \mathbf{A}+\mathbf{A} \circ(\nabla \mathbf{u})+(\nabla \mathbf{u})^{t} \circ \mathbf{A}+\alpha_{2} \mathbf{A} \circ \mathbf{A}\right) .
$$

We have used the notation $\circ$ for tensor multiplication, which here will be just matrix multiplication.

Thus the equations of motion (2.1) can be written

$$
\begin{aligned}
-\nu \Delta \mathbf{u}+\mathbf{u} \cdot \nabla \mathbf{u}+\nabla p & =\nabla \cdot \widehat{\boldsymbol{\tau}}, \\
\nabla \cdot \mathbf{u}=0 \quad \text { in } \Omega, \quad \mathbf{u} & =\mathbf{g} \quad \text { on } \partial \Omega .
\end{aligned}
$$

Here

$$
\begin{aligned}
\widehat{\boldsymbol{\tau}} & =\mathbf{T}_{\mathrm{G}}-\nu \mathbf{A}=\alpha_{1}\left(\mathbf{u} \cdot \nabla \mathbf{A}+\mathbf{A} \circ(\nabla \mathbf{u})+(\nabla \mathbf{u})^{t} \circ \mathbf{A}\right)+\alpha_{2} \mathbf{A} \circ \mathbf{A} \\
& =\alpha_{1}\left(\mathbf{u} \cdot \nabla \mathbf{A}-\mathbf{A} \circ(\nabla \mathbf{u})^{t}-(\nabla \mathbf{u}) \circ \mathbf{A}\right)+\left(2 \alpha_{1}+\alpha_{2}\right) \mathbf{A} \circ \mathbf{A} .
\end{aligned}
$$

We assume that the boundary data $\mathbf{g}$ is defined on all $\Omega$, is divergence free, and sufficiently smooth, to be specified subsequently.

\subsection{Solving the grade-two model equations}

It is helpful to expand the divergence of $\widehat{\boldsymbol{\tau}}$, defined in (2.4), to get a better sense of what the various terms are in (2.3). Recall (3.2) of [16] that

$$
\nabla \cdot(\mathbf{u} \cdot \nabla \mathbf{T})=\nabla \cdot\left(\mathbf{T} \circ(\nabla \mathbf{u})^{t}\right)+\mathbf{u} \cdot \nabla(\nabla \cdot \mathbf{T})
$$

for any tensor $\mathbf{T}$. Therefore

$$
\nabla \cdot(\mathbf{u} \cdot \nabla \mathbf{A})=\nabla \cdot\left(\mathbf{A} \circ(\nabla \mathbf{u})^{t}\right)+\mathbf{u} \cdot \nabla(\Delta \mathbf{u}) .
$$

Recall that $\mathbf{A}$ is shorthand for $\mathbf{A}=\nabla \mathbf{u}+\nabla \mathbf{u}^{t}$. Thus

$$
\begin{aligned}
\nabla \cdot \widehat{\boldsymbol{\tau}} & =\nabla \cdot\left(\alpha_{1}\left(\mathbf{A} \circ(\nabla \mathbf{u})^{t}+\mathbf{A} \circ(\nabla \mathbf{u})+(\nabla \mathbf{u})^{t} \circ \mathbf{A}\right)+\alpha_{2} \mathbf{A} \circ \mathbf{A}\right)+\alpha_{1} \mathbf{u} \cdot \nabla(\Delta \mathbf{u}) \\
& =\nabla \cdot\left(\alpha_{1}(\nabla \mathbf{u})^{t} \circ \mathbf{A}+\left(\alpha_{1}+\alpha_{2}\right) \mathbf{A} \circ \mathbf{A}\right)+\alpha_{1} \mathbf{u} \cdot \nabla(\Delta \mathbf{u}) .
\end{aligned}
$$

Equation (2.3) can thus be transformed [1] using (2.5):

$$
-\nu \Delta \mathbf{u}+\mathbf{u} \cdot \nabla \mathbf{u}+\nabla p-\alpha_{1} \mathbf{u} \cdot \nabla(\Delta \mathbf{u})=\nabla \cdot \widetilde{\boldsymbol{\tau}}
$$

where

$$
\tilde{\boldsymbol{\tau}}=\alpha_{1}(\nabla \mathbf{u})^{t} \circ \mathbf{A}+\left(\alpha_{1}+\alpha_{2}\right) \mathbf{A} \circ \mathbf{A} .
$$


Define the tensor $\mathbf{u} \otimes \mathbf{u}$ by $(\mathbf{u} \otimes \mathbf{u})_{i j}=u_{i} u_{j}$. Then

$$
\nabla \cdot(\mathbf{u} \otimes \mathbf{u})=\mathbf{u} \cdot \nabla \mathbf{u}
$$

and so (2.6) can be further transformed to

$$
-\nu \Delta \mathbf{u}-\alpha_{1} \mathbf{u} \cdot \nabla(\Delta \mathbf{u})+\nabla p=\nabla \cdot \boldsymbol{\tau},
$$

where

$$
\boldsymbol{\tau}=\widetilde{\boldsymbol{\tau}}-\mathbf{u} \otimes \mathbf{u}=\alpha_{1}(\nabla \mathbf{u})^{t} \circ \mathbf{A}+\left(\alpha_{1}+\alpha_{2}\right) \mathbf{A} \circ \mathbf{A}-\mathbf{u} \otimes \mathbf{u} .
$$

Thus

$$
\begin{aligned}
\mathbf{T}_{\mathrm{G}} & =\nu \mathbf{A}+\boldsymbol{\tau}+\alpha_{1}(\mathbf{u} \cdot \nabla \mathbf{A}+\mathbf{A} \circ(\nabla \mathbf{u})-\mathbf{A} \circ \mathbf{A})+\mathbf{u} \otimes \mathbf{u} \\
& =\nu \mathbf{A}+\boldsymbol{\tau}+\alpha_{1}\left(\mathbf{u} \cdot \nabla \mathbf{A}-\mathbf{A} \circ(\nabla \mathbf{u})^{t}\right)+\mathbf{u} \otimes \mathbf{u} .
\end{aligned}
$$

Note that $\boldsymbol{\tau}$ appears at first not to be a symmetric tensor due to the term $\nabla \mathbf{u}^{t} \circ \mathbf{A}$. However, in two dimensions this is a symmetric matrix if $\nabla \cdot \mathbf{u}=0$.

Lemma 2.1. Suppose that $M$ is a $2 \times 2$ matrix with trace zero. Then $M\left(M+M^{t}\right)$ is symmetric.

Proof. Write $M$ as

$$
M=\left(\begin{array}{cc}
a & b \\
c & -a
\end{array}\right)
$$

using the fact that the trace of $M$ is zero. Then

$$
\begin{aligned}
M\left(M+M^{t}\right) & =\left(\begin{array}{cc}
a & b \\
c-a
\end{array}\right)\left(\begin{array}{cc}
2 a & b+c \\
b+c & -2 a
\end{array}\right)=\left(\begin{array}{c}
2 a^{2}+b(b+c) a(b+c)-2 a b \\
2 a c-a(b+c) c(b+c)+2 a^{2}
\end{array}\right) \\
& =\left(\begin{array}{cc}
2 a^{2}+b c+b^{2} & a c-a b \\
a c-a b & 2 a^{2}+b c+c^{2}
\end{array}\right) .
\end{aligned}
$$

The latter matrix is evidently symmetric.

The lemma is not true for $3 \times 3$ matrices as simple examples show.

\section{TRANSFORMED GRADE-TWO MODEL EQUATIONS}

Let $\pi$ be defined by solving

$$
\nu \pi+\alpha_{1} \mathbf{u} \cdot \nabla \pi=p
$$

with suitable inflow boundary conditions $[5,8]$. Then

$$
\nabla p=\nu \nabla \pi+\alpha_{1}\left(\mathbf{u} \cdot \nabla(\nabla \pi)+\nabla \mathbf{u}^{t} \nabla \pi\right) .
$$

This means that

$$
\nabla p=\left(\nu I+\alpha_{1} \mathbf{u} \cdot \nabla\right) \nabla \pi+\alpha_{1} \nabla \mathbf{u}^{t} \nabla \pi
$$

Thus (2.9) transforms to

$$
\left(\nu I+\alpha_{1} \mathbf{u} \cdot \nabla\right)(-\Delta \mathbf{u}+\nabla \pi)+\alpha_{1} \nabla \mathbf{u}^{t} \nabla \pi=\nabla \cdot \boldsymbol{\tau} .
$$

Define

$$
N(\mathbf{u}, \pi)=-\alpha_{1} \pi \nabla \mathbf{u}^{t}+\boldsymbol{\tau}=-\alpha_{1} \pi \nabla \mathbf{u}^{t}+\alpha_{1}(\nabla \mathbf{u})^{t} \circ \mathbf{A}+\left(\alpha_{1}+\alpha_{2}\right) \mathbf{A} \circ \mathbf{A}-\mathbf{u} \otimes \mathbf{u}
$$


Note that $N$ is not a symmetric tensor due to the term $\pi \nabla \mathbf{u}^{t}$. The incompressibility condition $\nabla \cdot \mathbf{u}=0$ implies that

$$
\nabla \cdot\left(\pi \nabla \mathbf{u}^{t}\right)=\nabla \mathbf{u}^{t} \nabla \pi, \quad \nabla \cdot N(\mathbf{u}, \pi)=-\alpha_{1} \nabla \mathbf{u}^{t} \nabla \pi+\nabla \cdot \boldsymbol{\tau}
$$

Therefore

$$
\nabla \cdot N(\mathbf{u}, \pi)=-\alpha_{1} \nabla \mathbf{u}^{t} \nabla \pi+\nabla \cdot\left(\alpha_{1} \nabla \mathbf{u}^{t} \circ \mathbf{A}+\left(\alpha_{1}+\alpha_{2}\right) \mathbf{A} \circ \mathbf{A}-\mathbf{u} \otimes \mathbf{u}\right) .
$$

Thus (3.3) simplifies to

$$
\left(\nu I+\alpha_{1} \mathbf{u} \cdot\right)(-\Delta \mathbf{u}+\nabla \pi)=\nabla \cdot N(\mathbf{u}, \pi) .
$$

Now consider a coupled system that looks initially like a problem potentially different from (2.3), which is a slight variant of the one proposed in [1]:

$$
\begin{array}{lll}
-\Delta \mathbf{u}+\nabla \pi=\mathbf{w} \quad \text { in } \Omega, \quad \nabla \cdot \mathbf{u}=0 \quad \text { in } \Omega, \quad \mathbf{u}=\mathbf{g} & \text { on } \partial \Omega, \\
\left(\nu I+\alpha_{1} \mathbf{u} \cdot \nabla\right) \mathbf{w}=\nabla \cdot N(\mathbf{u}, \pi) & \text { in } \Omega, \quad \mathbf{w}=\mathbf{w}_{b} & \text { on } \Gamma_{-},
\end{array}
$$

where

$$
\Gamma_{-}=\left\{\mathbf{x} \in \partial \Omega \mid \alpha_{1} \mathbf{g}(\mathbf{x}) \cdot \mathbf{n}<0\right\} .
$$

Much of the paper will be devoted to proving this system is well posed and provides an equivalent formulation for solution of (2.3).

Theorem 3.1. The solution $(\mathbf{u}, \pi)$ of (3.8) satisfies (3.3). With p given by (3.1), then (u, $p$ ) satisfies (2.3) with $\widehat{\boldsymbol{\tau}}$ defined by (2.4). The vector function $\mathbf{w}$ satisfies

$$
\mathbf{w}=\frac{1}{\nu}(\nabla \cdot \widehat{\boldsymbol{\tau}}-\mathbf{u} \cdot \nabla \mathbf{u}-\nabla p)+\nabla \pi
$$

Proof. If $(\mathbf{u}, \pi)$ solves (3.8), then (3.7) holds. In view of (3.5), (3.3) then follows. Assuming $p$ solves (3.1), then $\nabla p$ satisfies (3.2). Combining (3.2) and (3.3), we get

$$
\left(\nu I+\alpha_{1} \mathbf{u} \cdot \nabla\right)(-\Delta \mathbf{u})+\nabla p=\nabla \cdot \boldsymbol{\tau},
$$

which is the same as (2.9). Reversing the derivation of (2.9) proves $(\mathbf{u}, p)$ satisfies (2.3) with $\widehat{\boldsymbol{\tau}}$ defined by (2.4).

The statement about $\mathbf{w}$ just involves replacing $-\Delta \mathbf{u}$ in (2.3) by the indicated expressions.

The difference between (3.8) and equation (2.6) of [1] is that $\mathbf{w}$ replaces $\nabla \cdot \sigma$ for a certain tensor $\sigma$, and a transport equation is posed for the full tensor $\sigma$ as opposed to the vector $\mathbf{w}$. Using (3.8) gives a smaller system to solve. The issue of inflow boundary conditions $[6,7]$ did not arise in [1] which was restricted to tangential flows. Thus in the general case, some suitable expression for $\sigma$ on the inflow boundary would be required.

\subsection{An algorithm for the transformed equations}

The system (3.8) is analogous to the reduced system in [15], and the algorithm in that paper suggests an algorithm for solving (3.8): start with some $\mathbf{w}^{0}$, then solve for $n \geq 1$

$$
\begin{array}{llll}
-\Delta \mathbf{u}^{n}+\nabla \pi^{n}=\mathbf{w}^{n-1} & \text { in } \Omega, \quad \nabla \cdot \mathbf{u}^{n}=0 & \text { in } \Omega, \quad \mathbf{u}^{n}=\mathbf{g} & \text { on } \partial \Omega, \\
\left(\nu I+\alpha_{1} \mathbf{u}^{n} \cdot \nabla\right) \mathbf{w}^{n}=\nabla \cdot N\left(\mathbf{u}^{n}, \pi^{n}\right) & \text { in } \Omega, & \mathbf{w}^{n}=\mathbf{w}_{b} & \text { on } \Gamma_{-} .
\end{array}
$$

For definiteness, we will take $\mathbf{w}^{0}=\mathbf{w}_{b}$. We prove convergence of this iteration for small data $\left(\mathbf{g}\right.$ and $\left.\mathbf{w}_{b}\right)$ in Section 4.3. To begin with, let us establish a basic bound.

We collect details on the Lebesgue and Sobolev spaces and norms used in Appendix A. Consider the Sobolev inequalities

$$
\|\mathbf{u}\|_{W_{\infty}^{1}(\Omega)} \leq \sigma_{q} \begin{cases}\|\mathbf{u}\|_{W_{q}^{2}(\Omega)}, & q>d, \\ \|\mathbf{u}\|_{W_{q}^{3}(\Omega)}, & q>d / 2(q \geq 1 \text { if } d=2) .\end{cases}
$$


Lemma 3.2. The operator in (3.6) is a continuous map

$$
\nabla \cdot N: W_{q}^{2}(\Omega)^{d} \times W_{q}^{1}(\Omega) \rightarrow L^{q}(\Omega)^{d}
$$

provided $q>d$. More precisely,

$$
\|\nabla \cdot N(\mathbf{u}, \pi)\|_{L^{q}(\Omega)} \leq C_{N}\|\mathbf{u}\|_{W_{q}^{2}(\Omega)}\left(\|\mathbf{u}\|_{W_{q}^{2}(\Omega)}+\|\pi\|_{W_{q}^{1}(\Omega)}\right),
$$

where $C_{N} \leq c \sigma_{q}\left(1+\left|\alpha_{1}\right|+\left|\alpha_{1}+\alpha_{2}\right|\right)$, $c$ is a constant that depends only on the dimension $d$, and $\sigma_{q}$ is the Sobolev constant in (3.10).

Proof. Applying (3.10) to (3.6), we get

$$
\|\nabla \cdot N(\mathbf{u}, \pi)\|_{L^{q}(\Omega)} \leq \sigma_{q}\left|\alpha_{1}\right|\|\mathbf{u}\|_{W_{q}^{2}(\Omega)}\|\pi\|_{W_{q}^{1}(\Omega)}+c\left\|\alpha_{1}(\nabla \mathbf{u})^{t} \circ \mathbf{A}+\left(\alpha_{1}+\alpha_{2}\right) \mathbf{A} \circ \mathbf{A}-\mathbf{u} \otimes \mathbf{u}\right\|_{W_{q}^{1}(\Omega)}
$$

for a constant $c$ that depends only on the dimension $d$. Note that

$$
\begin{aligned}
\left\|(\nabla \mathbf{u})^{t} \circ \mathbf{A}\right\|_{W_{q}^{1}(\Omega)}= & \left\|(\nabla \mathbf{u})^{t} \circ\left(\nabla \mathbf{u}+(\nabla \mathbf{u})^{t}\right)\right\|_{W_{q}^{1}(\Omega)} \\
\leq & \left\|(\nabla \mathbf{u})^{t} \circ\left(\nabla \mathbf{u}+(\nabla \mathbf{u})^{t}\right)\right\|_{L^{q}(\Omega)}+\left\|\nabla\left((\nabla \mathbf{u})^{t} \circ\left(\nabla \mathbf{u}+(\nabla \mathbf{u})^{t}\right)\right)\right\|_{L^{q}(\Omega)} \\
\leq & \left\|(\nabla \mathbf{u})^{t}\right\|_{L^{\infty}(\Omega)}\left\|\nabla \mathbf{u}+(\nabla \mathbf{u})^{t}\right\|_{L^{q}(\Omega)} \\
& +\left\|\nabla\left((\nabla \mathbf{u})^{t}\right) \circ\left(\nabla \mathbf{u}+(\nabla \mathbf{u})^{t}\right)\right\|_{L^{q}(\Omega)}+\left\|(\nabla \mathbf{u})^{t} \circ \nabla\left(\nabla \mathbf{u}+(\nabla \mathbf{u})^{t}\right)\right\|_{L^{q}(\Omega)} \\
\leq & \|\mathbf{u}\|_{W_{\infty}^{1}(\Omega)} 2\|\mathbf{u}\|_{W_{q}^{1}(\Omega)}+\|\mathbf{u}\|_{W_{q}^{2}(\Omega)} 2\|\mathbf{u}\|_{W_{\infty}^{1}(\Omega)}+\|\mathbf{u}\|_{W_{\infty}^{1}(\Omega)} 2\|\mathbf{u}\|_{W_{q}^{2}(\Omega)} .
\end{aligned}
$$

Thus (3.10) implies

$$
\left\|(\nabla \mathbf{u})^{t} \circ \mathbf{A}\right\|_{W_{q}^{1}(\Omega)} \leq 6 \sigma_{q}\|\mathbf{u}\|_{W_{q}^{2}(\Omega)}^{2}
$$

Similarly,

$$
\|\mathbf{A} \circ \mathbf{A}\|_{W_{q}^{1}(\Omega)}=\left\|\left(\nabla \mathbf{u}+(\nabla \mathbf{u})^{t}\right) \circ\left(\nabla \mathbf{u}+(\nabla \mathbf{u})^{t}\right)\right\|_{W_{q}^{1}(\Omega)} \leq 12 \sigma_{q}\|\mathbf{u}\|_{W_{q}^{2}(\Omega)}^{2} .
$$

Finally,

$$
\begin{aligned}
\|\mathbf{u} \otimes \mathbf{u}\|_{W_{q}^{1}(\Omega)} & \leq\|\mathbf{u} \otimes \mathbf{u}\|_{L^{q}(\Omega)}+\|\nabla(\mathbf{u} \otimes \mathbf{u})\|_{L^{q}(\Omega)} \leq 3\|\mathbf{u}\|_{L^{\infty}(\Omega)}\|\mathbf{u}\|_{W_{q}^{1}(\Omega)} \\
& \leq 3 \sigma_{q}\|\mathbf{u}\|_{W_{q}^{1}(\Omega)}^{2} \leq 3 \sigma_{q}\|\mathbf{u}\|_{W_{q}^{2}(\Omega)}^{2}
\end{aligned}
$$

Combining these estimates yields (3.11).

Lemma 3.3. The operator in (3.6) is a continuous map

$$
\nabla \cdot N: W_{q}^{3}(\Omega)^{d} \times W_{q}^{2}(\Omega) \rightarrow W_{q}^{1}(\Omega)^{d}
$$

provided $q>d / 2(q \geq 1$ if $d=2)$. Moreover

$$
\|\nabla \nabla \cdot N(\mathbf{u}, \pi)\|_{L^{q}(\Omega)} \leq C_{N}\|\mathbf{u}\|_{W_{q}^{3}(\Omega)}\left(\|\mathbf{u}\|_{W_{q}^{3}(\Omega)}+\|\pi\|_{W_{q}^{2}(\Omega)}\right),
$$

where $C_{N} \leq c \sigma_{q}\left(1+\left|\alpha_{1}\right|+\left|\alpha_{1}+\alpha_{2}\right|\right)$, $c$ is a constant that depends only on the dimension $d$, and $\sigma_{q}$ is the Sobolev constant in (3.10). 
Proof. One consequence of the assumption $q>d / 2$ is the Sobolev inequality

$$
\|\mathbf{T} \mathbf{U}\|_{L^{q}(\Omega)} \leq \sigma_{q}^{\prime}\|\mathbf{T}\|_{W_{q}^{1}(\Omega)}\|\mathbf{U}\|_{W_{q}^{1}(\Omega)}
$$

for any tensors $\mathbf{T}, \mathbf{U}$.

In view of $(3.6)$, we get

$$
\|\nabla \nabla \cdot N(\mathbf{u}, \pi)\|_{L^{q}(\Omega)} \leq\left|\alpha_{1}\right|\left\|\nabla\left(\nabla \mathbf{u}^{t} \nabla \pi\right)\right\|_{L^{q}(\Omega)}+\left\|\nabla \nabla \cdot\left(\alpha_{1}(\nabla \mathbf{u})^{t} \circ \mathbf{A}+\left(\alpha_{1}+\alpha_{2}\right) \mathbf{A} \circ \mathbf{A}-\mathbf{u} \otimes \mathbf{u}\right)\right\|_{L^{q}(\Omega)} .
$$

We have from the Sobolev inequalities (3.13) and (3.10) that

$$
\left\|\nabla\left(\nabla \mathbf{u}^{t} \nabla \pi\right)\right\|_{L^{q}(\Omega)} \leq\left\|\nabla^{2} \mathbf{u}^{t} \nabla \pi\right\|_{L^{q}(\Omega)}+\left\|\nabla \mathbf{u}^{t} \nabla^{2} \pi\right\|_{L^{q}(\Omega)} \leq\left(\sigma_{q}^{\prime}+\sigma_{q}\right)\|\mathbf{u}\|_{W_{q}^{3}(\Omega)}\|\|_{W_{q}^{2}(\Omega)} .
$$

Similarly, for a constant $c$ that depends only on the dimension $d$, we have

$$
\|\nabla \nabla \cdot(\mathbf{A} \circ \mathbf{A})\|_{L^{q}(\Omega)} \leq 2\left(\|(\nabla \mathbf{A}) \nabla \cdot \mathbf{A}\|_{L^{q}(\Omega)}+\|(\nabla \nabla \cdot \mathbf{A}) \mathbf{A}\|_{L^{q}(\Omega)}\right) \leq c\left(\sigma_{q}^{\prime}+\sigma_{q}\right)\|\mathbf{u}\|_{W_{q}^{3}(\Omega)}^{2} .
$$

The remaining terms are similar.

We can recover the physical pressure $p$ from (3.1), that is

$$
p=\nu \pi+\alpha_{1} \mathbf{u} \cdot \nabla \pi .
$$

One computational challenge is that (2.3) is a third-order PDE due to the presence of the term $\mathbf{u} \cdot \nabla(\Delta \mathbf{u})$. Thus we need to be careful about the number of boundary conditions required to get a unique solution.

\subsection{Variational formulation}

A variational formulation of (3.9) is as follows. The first two equations can be approximated by the iterated penalty method: find $\mathbf{u}^{n, \ell} \in V_{h}+\mathbf{g}$ such that

$$
\begin{aligned}
\int_{\Omega} \nabla \mathbf{u}^{n, \ell}: \nabla \mathbf{v} \mathrm{d} x+\rho \int_{\Omega} \nabla \cdot \mathbf{u}^{n, \ell} \nabla \cdot \mathbf{v} \mathrm{d} x & =\int_{\Omega} \mathbf{w}^{n-1} \cdot \mathbf{v} \mathrm{d} x-\int_{\Omega} \nabla \cdot \mathbf{z}^{\ell} \nabla \cdot \mathbf{v} \mathrm{d} x \quad \forall \mathbf{v} \in V_{h}, \\
\mathbf{z}^{\ell+1} & =\mathbf{z}^{\ell}+\rho \mathbf{u}^{n, \ell} .
\end{aligned}
$$

Once this is converged, we set $\mathbf{u}^{n}=\mathbf{u}^{n, \ell}$ and define the pressure via [22]

$$
\int_{\Omega} \pi^{n} q \mathrm{~d} x=\int_{\Omega}-\nabla \cdot \mathbf{z}^{\ell+1} q \mathrm{~d} x \quad \forall q \in \Pi_{h} .
$$

Note that $\pi^{n}$ has mean zero if constant functions are in $\Pi_{h}$, in view of the divergence theorem:

$$
\int_{\Omega} \pi^{n} \mathrm{~d} x=\int_{\Omega}-\nabla \cdot \mathbf{z}^{\ell+1} \mathrm{~d} x=-\int_{\partial \Omega} \mathbf{n} \cdot \mathbf{z}^{\ell+1} \mathrm{~d} s=c \int_{\partial \Omega} \mathbf{n} \cdot \mathbf{g} \mathrm{d} s=0 .
$$

We can pose the transport equation (3.9) via: find $\mathbf{w}^{n} \in \widetilde{V}_{h}+\mathbf{w}_{b}$ such that

$$
\nu \int_{\Omega} \mathbf{w}^{n} \cdot \mathbf{v} \mathrm{d} x+\alpha_{1} \int_{\Omega}\left(\mathbf{u}^{n} \cdot \nabla \mathbf{w}^{n}\right) \cdot \mathbf{v} \mathrm{d} x-\int_{\Omega}\left(\nabla \cdot N\left(\mathbf{u}^{n}, \pi^{n}\right)\right) \cdot \mathbf{v} \mathrm{d} x=0 \quad \forall \mathbf{v} \in \widetilde{V}_{h},
$$

where $\mathbf{w}_{b}$ is posed only on the inflow boundary, that is,

$$
\widetilde{V}_{h}=\left\{\mathbf{v} \in W_{h} \mid \mathbf{v}=\mathbf{0} \text { on } \Gamma_{-}\right\}, \quad \Gamma_{-}=\{\mathbf{x} \in \partial \Omega \mid \mathbf{n} \cdot \mathbf{g}<0\},
$$


whereas

$$
V_{h}=\left\{\mathbf{v} \in W_{h} \mid \mathbf{v}=\mathbf{0} \text { on } \partial \Omega\right\} .
$$

Note that it is tempting to integrate by parts to get

$$
\nu \int_{\Omega} \mathbf{w}^{n} \cdot \mathbf{v} \mathrm{d} x+\alpha_{1} \int_{\Omega}\left(\mathbf{u}^{n} \cdot \nabla \mathbf{w}^{n}\right) \cdot \mathbf{v} \mathrm{d} x+\int_{\Omega} N\left(\mathbf{u}^{n}, \pi^{n}\right): \nabla \mathbf{v} \mathrm{d} x=\ldots \quad \forall \mathbf{v} \in \widetilde{V}_{h},
$$

but there would be boundary terms that would need to be added to the formulation.

We can take $W_{h}$ to be continuous, vector-valued, piecewise polynomials of degree $k$ and $\Pi_{h}$ to be continuous, scalar-valued, piecewise polynomials of degree $k-1$. The use of continuous elements in (3.16) is called the unified Stokes algorithm (USA) [22].

Recall from (3.6) that

$$
\nabla \cdot N(\mathbf{u}, \pi)=-\alpha_{1} \nabla \mathbf{u}^{t} \nabla \pi+\nabla \cdot\left(\alpha_{1}(\nabla \mathbf{u})^{t} \circ \mathbf{A}+\left(\alpha_{1}+\alpha_{2}\right) \mathbf{A} \circ \mathbf{A}-\mathbf{u} \otimes \mathbf{u}\right) .
$$

Recalling (2.8), we have

$$
\begin{aligned}
\int_{\Omega}\left(\nabla \cdot N\left(\mathbf{u}^{n}, \pi^{n}\right)\right) \cdot \mathbf{v} \mathrm{d} x= & \int_{\Omega}-\alpha_{1}\left(\left(\nabla \mathbf{u}^{n}\right)^{t} \nabla \pi^{n}\right) \cdot \mathbf{v} \mathrm{d} x \\
& +\int_{\Omega}\left(\nabla \cdot\left(\alpha_{1}\left(\nabla \mathbf{u}^{n}\right)^{t} \circ \mathbf{A}^{n}+\left(\alpha_{1}+\alpha_{2}\right) \mathbf{A}^{n} \circ \mathbf{A}^{n}\right)\right) \cdot \mathbf{v} \mathrm{d} x \\
& -\int_{\Omega}\left(\mathbf{u}^{n} \cdot \nabla \mathbf{u}^{n}\right) \cdot \mathbf{v} \mathrm{d} x, \quad \mathbf{A}^{n}=\left(\nabla \mathbf{u}^{n}\right)^{t}+\nabla \mathbf{u}^{n} .
\end{aligned}
$$

We can compute the physical pressure $p^{n}$ from (3.14) via

$$
\int_{\Omega} p^{n} q \mathrm{~d} x=\int_{\Omega}\left(\nu \pi^{n}+\alpha_{1} \mathbf{u} \cdot \nabla \pi^{n}\right) q \mathrm{~d} x \quad \forall q \in \Pi_{h},
$$

but this does not need to be done at each iteration.

\subsection{Required inflow boundary conditions}

One drawback to the proposed method (3.9) is that it requires specification of boundary conditions for $\mathbf{w}=-\Delta \mathbf{u}+\nabla \pi$. Although we cannot provide general guidance for this, we can compute boundary conditions for $\mathbf{w}$ for typical flow geometries. We present this in Appendix B.

\section{TheORETicAl Details}

Here we collect the theoretical details required to prove the validity of our algorithm. We begin with an assumption about the smoothness of the data and domain. First we assume that for some $q>d, \mathbf{g} \in W_{q}^{2}(\Omega)$, with

$$
\oint_{\partial \Omega} \mathbf{n} \cdot \mathbf{g} \mathrm{d} s=0 .
$$

Further, we assume that there is a constant $c_{q}$ such that for any $\mathbf{g}$ as above and any $\mathbf{w} \in L^{q}(\Omega)$ the solution $(\mathbf{u}, \pi)$ of

$$
-\Delta \mathbf{u}+\nabla \pi=\mathbf{w} \quad \text { and } \quad \nabla \cdot \mathbf{u}=0 \quad \text { in } \Omega, \quad \text { with } \mathbf{u}=\mathbf{g} \quad \text { on } \partial \Omega
$$

satisfies, for $s=0,1$,

$$
\|\mathbf{u}\|_{W_{q}^{s+2}(\Omega)}+\|\pi\|_{W_{q}^{s+1}(\Omega)} \leq c_{q}\left(\|\mathbf{w}\|_{W_{q}^{s}(\Omega)}+\|\mathbf{g}\|_{W_{q}^{s+2}(\Omega)}\right), \quad q \leq Q_{s},
$$


where $c_{q}$ depends on $\Omega$ as well as $q$. For $s=0$, we require $Q_{s}>d$, but for $s=1$ we only require $Q_{s}>d / 2$. This follows from Theorem 5.4 in [13], page 88 when $\partial \Omega$ is sufficiently smooth. In our computational tests, we will have less smoothness with the polyhedral domains used, but these could be approximated by smooth domains.

We will prove the following theorem which establishes the existence of solutions for the grade-two model (2.3) as well as for the equivalent model (3.8).

Theorem 4.1. Assume that (4.2) holds for the Stokes problem (4.1). Suppose that $d /(s+1)<q \leq Q_{s}$, for $s=0,1$, and that $r$ satisfies

$$
\frac{2}{d}>\frac{1}{r}>\frac{1}{q}+\frac{1}{2}
$$

Then there exist positive, finite constants $\gamma$ and $C_{w}$ such that if the boundary data satisfy

$$
\begin{aligned}
\left\|\mathbf{w}_{b}\right\|_{W_{q}^{1}(\Omega)}+\|\mathbf{g}\|_{W_{q}^{2}(\Omega)} & \leq \frac{1}{8 \gamma^{2}+2 \gamma}, \\
\left\|\mathbf{w}_{b}\right\|_{W_{r}^{2}(\Omega)} & \leq \frac{\nu}{(\nu+1) C_{w} \gamma}, \\
\text { and }\|\mathbf{g}\|_{W_{r}^{3}(\Omega)} & \leq \frac{1}{C_{w} \gamma},
\end{aligned}
$$

and the initial iterates are sufficiently small, then the iterates (3.9) are bounded for all $n>0$ :

$$
\left\|\mathbf{w}^{n}\right\|_{W_{q}^{s}(\Omega)} \leq \mathcal{K}, \quad\left\|\mathbf{u}^{n}\right\|_{W_{q}^{s+2}(\Omega)}+\left\|\pi^{n}\right\|_{W_{q}^{s+1}(\Omega)} \leq c_{q}\left(\|\mathbf{g}\|_{W_{q}^{s+2}(\Omega)}+\mathcal{K}\right),
$$

where $\mathcal{K}$ is a finite positive constant and $s=0,1$. Moreover, $\left(\mathbf{u}^{n}, \pi^{n}, \mathbf{w}^{n}\right)$ converge geometrically in $W_{r}^{2}(\Omega)^{d} \times$ $W_{r}^{1}(\Omega) \times L^{r}(\Omega)^{d}$ to the solution $(\mathbf{u}, \pi, \mathbf{w})$ of $(3.8)$, In view of Theorem 3.1, $(\mathbf{u}, p)$ is the solution of the grade-two model (2.3), where $p$ is related to $\pi$ by (3.1).

The constraint (4.3) implies $q>2$ for $d=2$ and $q>6$ for $d=3$, and thus the constraint $q>d$ is satisfied implicitly. In our computational experiments, we will see that the assumptions on the data size may not be very restrictive in practice.

\section{1. $L^{q}$ bounds on the iterates}

Applying (4.2) with $s=0$ to the algorithm (3.9), we have

$$
\left\|\mathbf{u}^{n}\right\|_{W_{q}^{2}(\Omega)}+\left\|\pi^{n}\right\|_{W_{q}^{1}(\Omega)} \leq c_{q}\left(\|\mathbf{g}\|_{W_{q}^{2}(\Omega)}+\left\|\mathbf{w}^{n-1}\right\|_{L^{q}(\Omega)}\right) .
$$

Consider the abstract transport problem

$$
\left(\nu I+\alpha_{1} \mathbf{u} \cdot \nabla\right) \mathbf{w}=\mathbf{f} \quad \text { in } \Omega, \quad \mathbf{w}=\mathbf{w}_{b} \quad \text { on } \Gamma_{-} .
$$

In [24], it is proved that this has a unique solution satisfying

$$
\nu\|\mathbf{w}\|_{L^{q}(\Omega)} \leq\|\mathbf{f}\|_{L^{q}(\Omega)}+(1+\nu)\left\|\mathbf{w}_{b}\right\|_{L^{q}(\Omega)}+\left|\alpha_{1}\right|\left\|\mathbf{u} \cdot \nabla \mathbf{w}_{b}\right\|_{L^{q}(\Omega)},
$$

where $2 \leq q \leq \infty$.

Applying (4.8), (3.11), and (4.6), we conclude that

$$
\begin{aligned}
\nu\left\|\mathbf{w}^{n}\right\|_{L^{q}(\Omega)} \leq & C_{N}\left\|\mathbf{u}^{n}\right\|_{W_{q}^{2}(\Omega)}\left(\left\|\mathbf{u}^{n}\right\|_{W_{q}^{2}(\Omega)}+\left\|\pi^{n}\right\|_{W_{q}^{1}(\Omega)}\right) \\
& +(1+\nu)\left\|\mathbf{w}_{b}\right\|_{L^{q}(\Omega)}+\left|\alpha_{1}\right|\left\|\mathbf{u}^{n} \cdot \nabla \mathbf{w}_{b}\right\|_{L^{q}(\Omega)} \\
\leq & C_{N} c_{q}\left\|\mathbf{u}^{n}\right\|_{W_{q}^{2}(\Omega)}\left(\|\mathbf{g}\|_{W_{q}^{2}(\Omega)}+\left\|\mathbf{w}^{n-1}\right\|_{L^{q}(\Omega)}\right) \\
& +(1+\nu)\left\|\mathbf{w}_{b}\right\|_{L^{q}(\Omega)}+\left|\alpha_{1}\right|\left\|\mathbf{u}^{n}\right\|_{W_{\infty}^{1}(\Omega)}\left\|\mathbf{w}_{b}\right\|_{W_{q}^{1}(\Omega)}
\end{aligned}
$$


Thus (3.10) implies

$$
\begin{aligned}
\nu\left\|\mathbf{w}^{n}\right\|_{L^{q}(\Omega)} \leq & C_{N} c_{q}\left\|\mathbf{u}^{n}\right\|_{W_{q}^{2}(\Omega)}\left(\|\mathbf{g}\|_{W_{q}^{2}(\Omega)}+\left\|\mathbf{w}^{n-1}\right\|_{L^{q}(\Omega)}\right) \\
& +(1+\nu)\left\|\mathbf{w}_{b}\right\|_{L^{q}(\Omega)}+\sigma_{q}\left|\alpha_{1}\right|\left\|\mathbf{u}^{n}\right\|_{W_{q}^{2}(\Omega)}\left\|\mathbf{w}_{b}\right\|_{W_{q}^{1}(\Omega)} \\
\leq & \left(c_{q} C_{N}\|\mathbf{g}\|_{W_{q}^{2}(\Omega)}+\sigma_{q}\left|\alpha_{1}\right|\left\|\mathbf{w}_{b}\right\|_{W_{q}^{1}(\Omega)}\right)\left\|\mathbf{u}^{n}\right\|_{W_{q}^{2}(\Omega)} \\
& +C_{N} c_{q}\left\|\mathbf{u}^{n}\right\|_{W_{q}^{2}(\Omega)}\left\|\mathbf{w}^{n-1}\right\|_{L^{q}(\Omega)}+(1+\nu)\left\|\mathbf{w}_{b}\right\|_{L^{q}(\Omega)} .
\end{aligned}
$$

Define $\omega_{n}=\left\|\mathbf{w}^{n}\right\|_{L^{q}(\Omega)}, \eta_{n}=\left\|\mathbf{u}^{n}\right\|_{W_{q}^{2}(\Omega)}$, and

$$
\epsilon=\max \left\{\nu^{-1}\left(c_{q} C_{N}\|\mathbf{g}\|_{W_{q}^{2}(\Omega)}+\sigma_{q}\left|\alpha_{1}\right|\left\|\mathbf{w}_{b}\right\|_{W_{q}^{1}(\Omega)}\right),(1+1 / \nu)\left\|\mathbf{w}_{b}\right\|_{W_{q}^{1}(\Omega)}, c_{q}\|\mathbf{g}\|_{W_{q}^{2}(\Omega)}\right\} .
$$

Let $C_{G}=C_{N} c_{q} / \nu$. Then we have proved that

$$
\begin{aligned}
\eta_{n} & \leq \epsilon+c_{q} \omega_{n-1} \\
\omega_{n} & \leq C_{G} \eta_{n} \omega_{n-1}+\epsilon\left(1+\eta_{n}\right)=\left(C_{G} \omega_{n-1}+\epsilon\right) \eta_{n}+\epsilon \\
& \leq\left(C_{G} \omega_{n-1}+\epsilon\right)\left(\epsilon+c_{q} \omega_{n-1}\right)+\epsilon=C_{G} c_{q} \omega_{n-1}^{2}+\epsilon\left(\left(C_{G}+c_{q}\right) \omega_{n-1}+1\right)+\epsilon^{2} .
\end{aligned}
$$

Define $\gamma$ to be any constant such that

$$
\gamma \geq \gamma_{0}=\max \left\{c_{q} C_{G}, c_{q}+C_{G}, 1\right\} .
$$

Then (4.9) implies

$$
\omega_{n} \leq \gamma \epsilon\left(1+\epsilon+\omega_{n-1}\right)+\gamma \omega_{n-1}^{2} .
$$

Choosing $\epsilon \leq 1 / 4 \gamma$, we conclude that

$$
\omega_{n} \leq \epsilon\left(\gamma+\frac{1}{4}\right)+\frac{1}{4} \omega_{n-1}+\gamma \omega_{n-1}^{2} .
$$

Thus if $\omega_{n-1} \leq \frac{1}{4 \gamma}$, then

Now choose

$$
\omega_{n} \leq \epsilon\left(\gamma+\frac{1}{4}\right)+\frac{1}{2} \omega_{n-1} \leq \epsilon\left(\gamma+\frac{1}{4}\right)+\frac{1}{8 \gamma} .
$$

$$
\epsilon=\frac{1}{8 \gamma^{2}+2 \gamma} .
$$

Then we conclude that $\omega_{n} \leq \frac{1}{4 \gamma}$ as well. Note that by definition, $\gamma \geq 1$, so $\epsilon \leq 1 / 4 \gamma$. Recall that we have taken $\mathbf{w}^{0}=\mathbf{w}_{b}$.

Therefore, if the boundary data is sufficiently small, e.g.,

$$
\left\|\mathbf{w}_{b}\right\|_{W_{q}^{1}(\Omega)}+\|\mathbf{g}\|_{W_{q}^{2}(\Omega)} \leq \frac{1}{8 \gamma^{2}+2 \gamma}
$$

we conclude that in particular that $\left\|\mathbf{w}^{0}\right\|_{L^{q}(\Omega)} \leq \frac{1}{4 \gamma}$, and thus

$$
\left\|\mathbf{w}^{n}\right\|_{L^{q}(\Omega)} \leq \frac{1}{4 \gamma}
$$

for all $n>0$. Thus also

$$
\left\|\mathbf{u}^{n}\right\|_{W_{q}^{2}(\Omega)}+\left\|\pi^{n}\right\|_{W_{q}^{1}(\Omega)} \leq c_{q}\left(\|\mathbf{g}\|_{W_{q}^{2}(\Omega)}+\frac{1}{4 \gamma}\right) \leq \frac{c_{q}}{2 \gamma}
$$

for all $n>0$. Note that we can take the constant $\gamma$ as large as we like. 


\section{2. $W_{q}^{1}$ bounds on the iterates}

Applying (4.2) with $s=1$ to the algorithm (3.9), we have

$$
\left\|\mathbf{u}^{n}\right\|_{W_{r}^{3}(\Omega)}+\left\|\pi^{n}\right\|_{W_{r}^{2}(\Omega)} \leq c_{r}\left(\|\mathbf{g}\|_{W_{r}^{3}(\Omega)}+\left\|\mathbf{w}^{n-1}\right\|_{W_{r}^{1}(\Omega)}\right) .
$$

In [24], it is proved that the unique solution of (4.7) satisfies

$$
\nu\|\mathbf{w}\|_{W_{r}^{1}(\Omega)} \leq\|\mathbf{f}\|_{W_{r}^{1}(\Omega)}+c_{q r}\|\mathbf{f}\|_{L^{q}(\Omega)}+(1+\nu)\left\|\mathbf{w}_{b}\right\|_{W_{r}^{1}(\Omega)}+\left|\alpha_{1}\right|\left\|\mathbf{u} \cdot \nabla \mathbf{w}_{b}\right\|_{W_{r}^{1}(\Omega)},
$$

where $1 \leq r<2$ and $q \leq \infty$ satisfies

$$
\frac{1}{q}<\frac{1}{r}-\frac{1}{2}
$$

Applying (4.15) with $\mathbf{f}=\nabla \cdot N\left(\mathbf{u}^{n}, \pi^{n}\right)$ as in (3.9), then (3.12), (3.11), (4.14), and (3.13) imply that

$$
\begin{aligned}
\nu\left\|\mathbf{w}^{n}\right\|_{W_{r}^{1}(\Omega)} \leq & C_{N}\left\|\mathbf{u}^{n}\right\|_{W_{r}^{3}(\Omega)}\left(\left\|\mathbf{u}^{n}\right\|_{W_{r}^{3}(\Omega)}+\left\|\pi^{n}\right\|_{W_{r}^{2}(\Omega)}\right) \\
& +C_{N} c_{q r}\left\|\mathbf{u}^{n}\right\|_{W_{q}^{2}(\Omega)}\left(\left\|\mathbf{u}^{n}\right\|_{W_{q}^{2}(\Omega)}+\left\|\pi^{n}\right\|_{W_{q}^{1}(\Omega)}\right) \\
& +(1+\nu)\left\|\mathbf{w}_{b}\right\|_{W_{r}^{1}(\Omega)}+\left|\alpha_{1}\right|\left\|\mathbf{u}^{n} \cdot \nabla \mathbf{w}_{b}\right\|_{W_{r}^{1}(\Omega)} \\
\leq & C_{N} c_{r}^{2}\left(\|\mathbf{g}\|_{W_{r}^{3}(\Omega)}+\left\|\mathbf{w}^{n-1}\right\|_{W_{r}^{1}(\Omega)}\right)^{2}+\frac{C_{N} c_{q}^{2} c_{q r}}{4 \gamma^{2}}+(1+\nu)\left\|\mathbf{w}_{b}\right\|_{W_{r}^{1}(\Omega)} \\
& +\left|\alpha_{1}\right|\left(\left\|\mathbf{u}^{n}\right\|_{L^{\infty}(\Omega)}\left\|\mathbf{w}_{b}\right\|_{W_{r}^{2}(\Omega)}+\sigma_{r}^{\prime}\left\|\mathbf{u}^{n}\right\|_{W_{r}^{2}(\Omega)}\left\|\mathbf{w}_{b}\right\|_{W_{r}^{2}(\Omega)}\right) .
\end{aligned}
$$

Note that Hölder's inequality and (4.13) imply

$$
\left\|\mathbf{u}^{n}\right\|_{W_{r}^{2}(\Omega)} \leq|\Omega|^{1-r / q}\left\|\mathbf{u}^{n}\right\|_{W_{q}^{2}(\Omega)} \leq|\Omega|^{1-r / q} \frac{c_{q}}{2 \gamma}
$$

Combining this with (4.16) yields

$$
\begin{aligned}
\nu\left\|\mathbf{w}^{n}\right\|_{W_{r}^{1}(\Omega)} \leq & C_{N} c_{r}^{2}\left(g_{3}+\left\|\mathbf{w}^{n-1}\right\|_{W_{r}^{1}(\Omega)}\right)^{2}+\frac{C_{N} c_{q}^{2} c_{q r}}{4 \gamma^{2}} \\
& +\left((1+\nu)+\frac{\left|\alpha_{1}\right|\left(\sigma_{q}+\sigma_{r}^{\prime}|\Omega|^{1-r / q}\right) c_{q}}{2 \gamma}\right)\left\|\mathbf{w}_{b}\right\|_{W_{r}^{2}(\Omega)},
\end{aligned}
$$

where $g_{3}=\|\mathbf{g}\|_{W_{r}^{3}(\Omega)}$, provided $\gamma$ satisfies (4.10). Define $C_{w}=\nu^{-1} C_{N} c_{r}^{2}$ and

$$
\epsilon=\frac{C_{N} c_{q}^{2} c_{q r}}{4 \nu \gamma^{2}}+\frac{1}{\nu}\left((1+\nu)+\frac{\left|\alpha_{1}\right|\left(\sigma_{q}+\sigma_{r}^{\prime}|\Omega|^{1-r / q}\right) c_{q}}{2 \gamma}\right)\left\|\mathbf{w}_{b}\right\|_{W_{r}^{2}(\Omega)} .
$$

Then (4.17) implies

$$
\left\|\mathbf{w}^{n}\right\|_{W_{r}^{1}(\Omega)} \leq C_{w}\left(g_{3}+\left\|\mathbf{w}^{n-1}\right\|_{W_{r}^{1}(\Omega)}\right)^{2}+\epsilon .
$$

Define $\omega^{n}=C_{w}\left\|\mathbf{w}^{n}\right\|_{W_{r}^{1}(\Omega)}$. Then we have

$$
\omega^{n} \leq\left(C_{w} g_{3}+\omega^{n-1}\right)^{2}+C_{w} \epsilon .
$$

Assume that

$$
\left\|\mathbf{w}_{b}\right\|_{W_{r}^{2}(\Omega)} \leq \frac{\nu}{(\nu+1) C_{w} \gamma}
$$


Then

$$
C_{w} \epsilon \leq \frac{C_{w} C_{N} c_{q}^{2} c_{q r}}{4 \nu \gamma^{2}}+\frac{1}{\gamma}+\frac{\left|\alpha_{1}\right|\left(\sigma_{q}+\sigma_{r}^{\prime}|\Omega|^{1-r / q}\right) c_{q}}{2(\nu+1) \gamma^{2}} .
$$

By taking $\gamma$ sufficiently large, we have

$$
C_{w} \epsilon \leq \frac{2}{\gamma}
$$

More precisely, this holds when

$$
\gamma \geq \gamma_{1}=\max \left\{\gamma_{0}, \frac{C_{w} C_{N} c_{r}^{2} c_{q r}}{4 \nu}+\frac{\left|\alpha_{1}\right|\left(\sigma_{q}+\sigma_{r}^{\prime}|\Omega|^{1-r / q}\right) c_{q}}{2(\nu+1)}\right\},
$$

where $\gamma_{0}$ is defined in (4.10). Assume further that

$$
g_{3}=\|\mathbf{g}\|_{W_{r}^{3}(\Omega)} \leq \frac{1}{C_{w} \gamma} .
$$

Then (4.18) and (4.20) imply that

$$
\omega^{n} \leq\left(\frac{1}{\gamma}+\omega^{n-1}\right)^{2}+\frac{2}{\gamma}
$$

Note that (4.19) implies that

$$
\omega^{0}=C_{w}\left\|\mathbf{w}^{0}\right\|_{W_{r}^{1}(\Omega)}=C_{w}\left\|\mathbf{w}_{b}\right\|_{W_{r}^{1}(\Omega)} \leq C_{w}\left\|\mathbf{w}_{b}\right\|_{W_{r}^{2}(\Omega)} \leq \frac{\nu}{(\nu+1) \gamma} \leq \frac{1}{\gamma} .
$$

Under the inductive hypothesis that

$$
\omega^{n-1} \leq \frac{3}{\gamma}
$$

then (4.23) implies that

$$
\omega^{n} \leq\left(\frac{4}{\gamma}\right)^{2}+\frac{2}{\gamma} \leq \frac{3}{\gamma}
$$

provided that $\gamma \geq 16$. Therefore (4.24) implies that

$$
\left\|\mathbf{w}^{n}\right\|_{W_{r}^{1}(\Omega)} \leq \frac{3}{C_{w} \gamma}=\frac{3 \nu}{C_{N} c_{r}^{2} \gamma}
$$

for all $n$, provided that

$$
\gamma \geq \gamma_{2}=\max \left\{\gamma_{1}, 16\right\}
$$

where $\gamma_{1}$ is defined in (4.21).

Using (4.14), we find

$$
\left\|\mathbf{u}^{n}\right\|_{W_{r}^{3}(\Omega)}+\left\|\pi^{n}\right\|_{W_{r}^{2}(\Omega)} \leq c_{r}\left(\|\mathbf{g}\|_{W_{r}^{3}(\Omega)}+\left\|\mathbf{w}^{n-1}\right\|_{W_{r}^{1}(\Omega)}\right) \leq \frac{C_{r}}{\gamma}
$$

for all $n>0$, under the assumptions (4.19) and (4.22), where

$$
C_{r}=\frac{c_{r}}{C_{w}}+\frac{3 \nu}{C_{N} c_{r}} .
$$




\subsection{Convergence estimates}

Recall the tensor $\boldsymbol{\tau}$ introduced in (2.10):

$$
\boldsymbol{\tau}=\alpha_{1}(\nabla \mathbf{u})^{t} \circ \mathbf{A}+\left(\alpha_{1}+\alpha_{2}\right) \mathbf{A} \circ \mathbf{A}-\mathbf{u} \otimes \mathbf{u} .
$$

Thus (3.4) and (3.6) imply

$$
\nabla \cdot N(\mathbf{u}, \pi)=-\alpha_{1} \nabla \mathbf{u}^{t} \nabla \pi+\nabla \cdot \boldsymbol{\tau} .
$$

To estimate terms involving $N$, note that for any two sequences $a^{n}$ and $b^{n}$,

$$
a^{n} b^{n}-a^{n-1} b^{n-1}=a^{n} b^{n}-a^{n} b^{n-1}+a^{n} b^{n-1}-a^{n-1} b^{n-1}=a^{n}\left(b^{n}-b^{n-1}\right)+\left(a^{n}-a^{n-1}\right) b^{n-1} .
$$

Thus (4.29) implies

$$
\begin{aligned}
\left\|\nabla \cdot\left(N\left(\mathbf{u}^{n}, \pi^{n}\right)-N\left(\mathbf{u}^{n-1}, \pi^{n-1}\right)\right)\right\|_{L^{r}(\Omega)} \leq & \left|\alpha_{1}\right|\left\|\nabla\left(\mathbf{u}^{n}-\mathbf{u}^{n-1}\right)^{t} \nabla \pi^{n}+\nabla\left(\pi^{n}-\pi^{n-1}\right)\left(\nabla \mathbf{u}^{n-1}\right)^{t}\right\|_{L^{r}(\Omega)} \\
& +\left|\alpha_{1}\right|\left\|\nabla\left(\mathbf{u}^{n}\right)^{t} \circ\left(\mathbf{A}^{n}-\mathbf{A}^{n-1}\right)+\nabla\left(\mathbf{u}^{n}-\mathbf{u}^{n-1}\right)^{t} \circ \mathbf{A}^{n-1}\right\|_{W_{r}^{1}(\Omega)} \\
& +\left|\alpha_{1}+\alpha_{2}\right|\left\|\left(\mathbf{A}^{n}+\mathbf{A}^{n-1}\right) \circ\left(\mathbf{A}^{n}-\mathbf{A}^{n-1}\right)\right\|_{W_{r}^{1}(\Omega)} \\
& +\left\|\mathbf{u}^{n} \otimes\left(\mathbf{u}^{n}-\mathbf{u}^{n-1}\right)+\left(\mathbf{u}^{n}-\mathbf{u}^{n-1}\right) \otimes \mathbf{u}^{n-1}\right\|_{W_{r}^{1}(\Omega)}
\end{aligned}
$$

We examine these four terms separately. First, (3.13), (4.27), (3.10), and (4.13) give

$$
\begin{aligned}
& \left\|\nabla\left(\mathbf{u}^{n}-\mathbf{u}^{n-1}\right)^{t} \nabla \pi^{n}+\nabla\left(\pi^{n}-\pi^{n-1}\right)\left(\nabla \mathbf{u}^{n-1}\right)^{t}\right\|_{L^{r}(\Omega)} \\
& \quad \leq \sigma_{r}^{\prime}\left\|\nabla\left(\mathbf{u}^{n}-\mathbf{u}^{n-1}\right)\right\|_{W_{r}^{1}(\Omega)}\left\|\nabla \pi^{n}\right\|_{W_{r}^{1}(\Omega)}+\left\|\nabla\left(\pi^{n}-\pi^{n-1}\right)\right\|_{L^{r}(\Omega)}\left\|\nabla \mathbf{u}^{n-1}\right\|_{L^{\infty}(\Omega)} \\
& \quad \leq \frac{\sigma_{r}^{\prime} C_{r}}{\gamma}\left\|\nabla\left(\mathbf{u}^{n}-\mathbf{u}^{n-1}\right)\right\|_{W_{r}^{1}(\Omega)}+\sigma_{q}\left\|\nabla\left(\pi^{n}-\pi^{n-1}\right)\right\|_{L^{r}(\Omega)}\left\|\mathbf{u}^{n-1}\right\|_{W_{q}^{2}(\Omega)} \\
& \quad \leq \frac{c_{r} \sigma_{q}}{\gamma}\left\|\mathbf{u}^{n}-\mathbf{u}^{n-1}\right\|_{W_{r}^{2}(\Omega)}+\frac{\sigma_{q} c_{q}}{2 \gamma}\left\|\pi^{n}-\pi^{n-1}\right\|_{W_{r}^{1}(\Omega)} .
\end{aligned}
$$

For the second term, there is a constant $C$ depending only on the dimension such that

$$
\begin{aligned}
& \left\|\nabla\left(\mathbf{u}^{n}\right)^{t} \circ\left(\mathbf{A}^{n}-\mathbf{A}^{n-1}\right)+\nabla\left(\mathbf{u}^{n}-\mathbf{u}^{n-1}\right)^{t} \circ \mathbf{A}^{n-1}\right\|_{W_{r}^{1}(\Omega)} \\
& \quad \leq C\left(\left\|\mathbf{u}^{n}\right\|_{W_{\infty}^{1}(\Omega)}+\left\|\mathbf{u}^{n-1}\right\|_{W_{\infty}^{1}(\Omega)}\right)\left\|\mathbf{u}^{n}-\mathbf{u}^{n-1}\right\|_{W_{r}^{2}(\Omega)} \\
& \quad \leq \frac{C c_{q} \sigma_{q}}{\gamma}\left\|\mathbf{u}^{n}-\mathbf{u}^{n-1}\right\|_{W_{r}^{2}(\Omega)},
\end{aligned}
$$

and a similar estimate holds for the third and fourth terms. Thus (4.30) shows that

$$
\begin{aligned}
\left\|\nabla \cdot\left(N\left(\mathbf{u}^{n}, \pi^{n}\right)-N\left(\mathbf{u}^{n-1}, \pi^{n-1}\right)\right)\right\|_{L^{r}(\Omega)} & \leq \frac{C}{\gamma}\left(\left\|\mathbf{u}^{n}-\mathbf{u}^{n-1}\right\|_{W_{r}^{2}(\Omega)}+\left\|\pi^{n}-\pi^{n-1}\right\|_{W_{r}^{1}(\Omega)}\right) \\
& \leq \frac{C c_{r}}{\gamma}\left\|\mathbf{w}^{n-1}-\mathbf{w}^{n-2}\right\|_{L^{r}(\Omega)} .
\end{aligned}
$$

At the last step, we utilized the fact that $\mathbf{u}^{n}-\mathbf{u}^{n-1}$ is zero on the boundary, so we could apply (4.2) directly. Define $\mathbf{e}=\mathbf{w}^{n}-\mathbf{w}^{n-1}$. Then from (3.9)

$$
\begin{aligned}
\nu \mathbf{e}+\alpha_{1} \mathbf{u}^{n} \cdot \nabla \mathbf{e} & =G-\alpha_{1}\left(\mathbf{u}^{n}-\mathbf{u}^{n-1}\right) \cdot \nabla \mathbf{w}^{n-1}, \\
G & =\nabla \cdot\left(N\left(\mathbf{u}^{n}, \pi^{n}\right)-N\left(\mathbf{u}^{n-1}, \pi^{n-1}\right)\right) .
\end{aligned}
$$


Sobolev's inequality implies that

$$
\|\mathbf{v}\|_{L^{\infty}(\Omega)} \leq c^{\prime}\|\mathbf{v}\|_{W_{r}^{2}(\Omega)}
$$

for any $r>d / 2(r \geq 1$ if $d=2)$. Applying (4.8), (4.31), (4.33), and (4.25) implies

$$
\begin{aligned}
\nu\left\|\mathbf{w}^{n}-\mathbf{w}^{n-1}\right\|_{L^{r}(\Omega)} & \leq \frac{C c_{r}}{\gamma}\left\|\mathbf{w}^{n-1}-\mathbf{w}^{n-2}\right\|_{L^{r}(\Omega)}+\left|\alpha_{1}\right|\left\|\mathbf{u}^{n}-\mathbf{u}^{n-1}\right\|_{L^{\infty}(\Omega)}\left\|\mathbf{w}^{n-1}\right\|_{W_{r}^{1}(\Omega)} \\
& \leq \frac{C c_{r}}{\gamma}\left\|\mathbf{w}^{n-1}-\mathbf{w}^{n-2}\right\|_{L^{r}(\Omega)}+\left|\alpha_{1}\right| c^{\prime}\left\|\mathbf{u}^{n}-\mathbf{u}^{n-1}\right\|_{W_{r}^{2}(\Omega)}\left\|\mathbf{w}^{n-1}\right\|_{W_{r}^{1}(\Omega)} \\
& \leq \frac{C c_{r}}{\gamma}\left\|\mathbf{w}^{n-1}-\mathbf{w}^{n-2}\right\|_{L^{r}(\Omega)}+\frac{3 \nu\left|\alpha_{1}\right| c^{\prime}}{C_{N} c_{r}^{2} \gamma}\left\|\mathbf{u}^{n}-\mathbf{u}^{n-1}\right\|_{W_{r}^{2}(\Omega)} .
\end{aligned}
$$

Applying (4.2) to (4.34), we get

$$
\left\|\mathbf{w}^{n}-\mathbf{w}^{n-1}\right\|_{L^{r}(\Omega)} \leq \frac{1}{\gamma}\left(\frac{C c_{r}}{\nu}+\frac{3\left|\alpha_{1}\right| c^{\prime}}{C_{N} c_{r}}\right)\left\|\mathbf{w}^{n-1}-\mathbf{w}^{n-2}\right\|_{L^{r}(\Omega)} .
$$

Taking $\gamma$ sufficiently large, that is,

$$
\gamma=\gamma_{3}=\max \left\{\gamma_{2}, \frac{2 C c_{r}}{\nu}+\frac{6\left|\alpha_{1}\right| c^{\prime}}{C_{N} c_{r}}\right\},
$$

where $\gamma_{2}$ is defined in (4.26), and correspondingly restricting the size of the data as in (4.11), (4.19), and (4.22), if necessary, we conclude from (4.35) and (4.36) that

$$
\left\|\mathbf{w}^{n}-\mathbf{w}^{n-1}\right\|_{L^{r}(\Omega)} \leq \frac{1}{2}\left\|\mathbf{w}^{n-1}-\mathbf{w}^{n-2}\right\|_{L^{r}(\Omega)} .
$$

Therefore the sequence $\mathbf{w}^{n}$ converges geometrically in $L^{r}(\Omega)^{d}$. Subtracting iterates in (3.9), we find

$$
\begin{aligned}
& -\Delta\left(\mathbf{u}^{n}-\mathbf{u}^{n-1}\right)+\nabla\left(\pi^{n}-\pi^{n-1}\right)=\mathbf{w}^{n-1}-\mathbf{w}^{n-2} \quad \text { in } \Omega, \\
& \nabla \cdot\left(\mathbf{u}^{n}-\mathbf{u}^{n-1}\right)=0 \quad \text { in } \Omega, \quad \mathbf{u}^{n}-\mathbf{u}^{n-1}=\mathbf{0} \quad \text { on } \partial \Omega \text {. }
\end{aligned}
$$

Thus (4.2) implies that

$$
\left\|\mathbf{u}^{n}-\mathbf{u}^{n-1}\right\|_{W_{r}^{2}(\Omega)}+\left\|\pi^{n}-\pi^{n-1}\right\|_{W_{r}^{1}(\Omega)} \leq c_{r}\left\|\mathbf{w}^{n-1}-\mathbf{w}^{n-2}\right\|_{L^{r}(\Omega)},
$$

and thus the sequence $\mathbf{u}^{n}$ converges geometrically in $W_{r}^{2}(\Omega)^{d}$ and the sequence $\pi^{n}$ converges geometrically in $W_{r}^{1}(\Omega)$. This completes the proof of Theorem 4.1.

\section{Computational experiments}

Here we explore computational techniques for implementing the grade-two algorithm. Table 1 presents results for the algorithm (3.9) for $\nu=\alpha_{1}=\alpha_{2}=1$, implemented using quadratic and cubic elements using the iteratedpenalty method (IPM) [9], on the unit square domain (B.1) with $L=1$ and boundary data (B.11) with $U=1$. The exact $\pi$ is quadratic as indicated in (B.8). Using piecewise degree $k$ elements for $V_{h}$ results in piecewise degree $k-1$ elements for the pressure approximation. Thus for $k=2$, the pressure approximation is only piecewise linear, and the approximation of $\pi$ dominates the overall errors. Table 1a indicates that the error $e_{\pi}$ is close to second order. But for $k=3$, the exact $\pi$ is in the pressure space, and we get essentially round-off error. Due to some sort of instability, the errors grow as the mesh size is reduced, but they are significantly smaller than for the case $k=2$. Figure 2 shows that there is a localized error that occurs in $w_{1}$ (which should be identically zero) near the corners of the inflow boundary. This pollutes the component $w_{2}$ (which is isolated in Tab. 1) and causes errors in $\mathbf{u}$ and $\pi$. Computations for $k=4$ yielded similar results as for the $k=3$ case.

The error for approximating $\mathbf{w}$ in $H^{1}$ are much worse than for other errors. But we know from Section 4.2 that the transport problem does not have uniform bounds in $H^{1}$, so the larger errors are not surprising. 
TABle 1. Grade-two simulations of Poiseuille flow in the domain (B.1) with $L=1$ and $\nu=1$ and boundary data (B.11) with $U=1$ using (a) piecewise quadratics for $V_{h}$ and (b) piecewise cubics for $V_{h}$. The mesh consisted of an $M \times M$ array of Malkus splits (squares divided into four triangles by the bisectors) [26]. The algorithm (3.9) was implemented using the iterated penalty method (3.15). The column "iters" indicates the number of iterations of (3.9). The pressure was computed via USA [22] as described in (3.16) with $\Pi_{h}$ begin continuous piecewise polynomials of degree $k-1$. Errors: $e_{\mathbf{u}}=\left\|\mathbf{u}-\mathbf{u}_{h}\right\|_{H^{1}}, e_{\pi}=\left\|\pi-\pi_{h}\right\|_{L^{2}}, e_{\mathbf{w}, H^{1}}=\left\|\mathbf{w}-\mathbf{w}_{h}\right\|_{H^{1}}, e_{w_{1}}=$ $\left\|w_{1}-w_{1 h}\right\|_{H^{1}}, e_{w_{2}}=\left\|w_{2}-w_{2 h}\right\|_{H^{1}}, e_{\mathbf{w}, L^{2}}\left\|\mathbf{w}-\mathbf{w}_{h}\right\|_{L^{2}}$.

\begin{tabular}{llllllllllll}
\hline \hline & $M$ & $U$ & $\alpha_{1}$ & $\alpha_{2}$ & iters & $e_{\mathbf{u}}$ & $e_{\pi}$ & $e_{\mathbf{w}, H^{1}}$ & $e_{w_{1}}$ & $e_{w_{2}}$ & $e_{\mathbf{w}, L^{2}}$ \\
\hline & 8 & 1.0 & 1.0 & 1.0 & 23 & $3.20 \mathrm{e}-03$ & $1.44 \mathrm{e}-02$ & $1.72 \mathrm{e}-01$ & $2.37 \mathrm{e}-02$ & $1.70 \mathrm{e}-01$ & $2.43 \mathrm{e}-02$ \\
& 16 & 1.0 & 1.0 & 1.0 & 29 & $1.15 \mathrm{e}-03$ & $4.65 \mathrm{e}-03$ & $1.36 \mathrm{e}-01$ & $1.70 \mathrm{e}-02$ & $1.35 \mathrm{e}-01$ & $1.29 \mathrm{e}-02$ \\
(a) & 32 & 1.0 & 1.0 & 1.0 & 37 & $3.48 \mathrm{e}-04$ & $1.38 \mathrm{e}-03$ & $1.02 \mathrm{e}-01$ & $1.18 \mathrm{e}-02$ & $1.01 \mathrm{e}-01$ & $5.82 \mathrm{e}-03$ \\
& 64 & 1.0 & 1.0 & 1.0 & 46 & $9.86 \mathrm{e}-05$ & $3.88 \mathrm{e}-04$ & $7.44 \mathrm{e}-02$ & $8.31 \mathrm{e}-03$ & $7.40 \mathrm{e}-02$ & $2.37 \mathrm{e}-03$ \\
& 128 & 1.0 & 1.0 & 1.0 & 58 & $2.76 \mathrm{e}-05$ & $1.07 \mathrm{e}-04$ & $5.35 \mathrm{e}-02$ & $5.87 \mathrm{e}-03$ & $5.32 \mathrm{e}-02$ & $9.11 \mathrm{e}-04$ \\
& 256 & 1.0 & 1.0 & 1.0 & 80 & $7.59 \mathrm{e}-06$ & $2.92 \mathrm{e}-05$ & $3.82 \mathrm{e}-02$ & $4.15 \mathrm{e}-03$ & $3.80 \mathrm{e}-02$ & $3.39 \mathrm{e}-04$ \\
\hline & 8 & 1.0 & 1.0 & 1.0 & 2 & $4.13 \mathrm{e}-10$ & $6.30 \mathrm{e}-10$ & $5.15 \mathrm{e}-08$ & $1.27 \mathrm{e}-08$ & $4.99 \mathrm{e}-08$ & $3.15 \mathrm{e}-09$ \\
& 16 & 1.0 & 1.0 & 1.0 & 2 & $8.75 \mathrm{e}-10$ & $1.89 \mathrm{e}-09$ & $3.71 \mathrm{e}-07$ & $4.17 \mathrm{e}-08$ & $3.69 \mathrm{e}-07$ & $1.17 \mathrm{e}-08$ \\
(b) & 32 & 1.0 & 1.0 & 1.0 & 2 & $2.03 \mathrm{e}-09$ & $6.97 \mathrm{e}-09$ & $2.89 \mathrm{e}-06$ & $1.52 \mathrm{e}-07$ & $2.89 \mathrm{e}-06$ & $4.61 \mathrm{e}-08$ \\
& 64 & 1.0 & 1.0 & 1.0 & 2 & $5.67 \mathrm{e}-09$ & $2.76 \mathrm{e}-08$ & $2.30 \mathrm{e}-05$ & $7.02 \mathrm{e}-07$ & $2.30 \mathrm{e}-05$ & $1.84 \mathrm{e}-07$ \\
& 128 & 1.0 & 1.0 & 1.0 & 3 & $2.09 \mathrm{e}-08$ & $1.03 \mathrm{e}-07$ & $1.88 \mathrm{e}-04$ & $4.39 \mathrm{e}-06$ & $1.88 \mathrm{e}-04$ & $7.59 \mathrm{e}-07$ \\
\hline
\end{tabular}
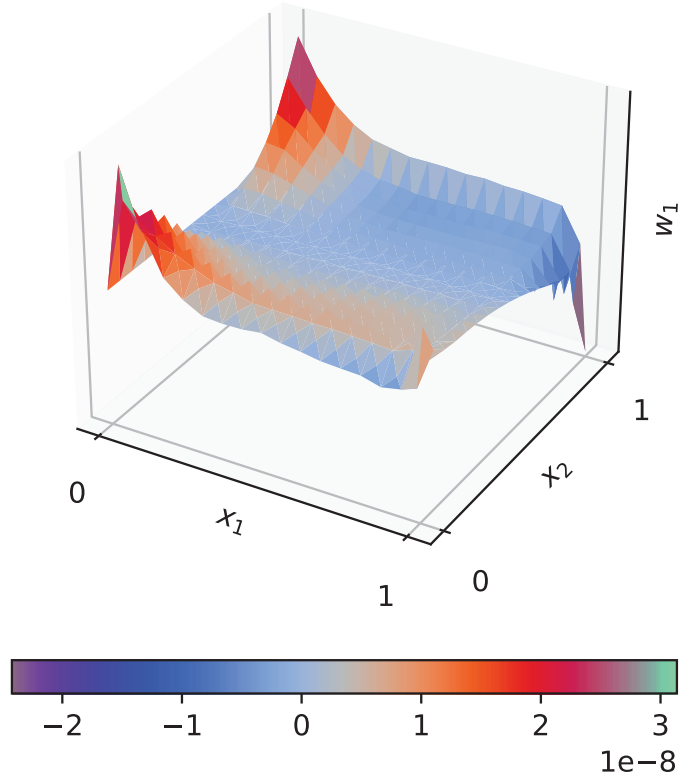

FIgURE 2. The component $w_{1}$ for the computations in Table $1 \mathrm{~b}$ with $M=16$.

\section{Conclusions}

We developed an algorithm for solving the general grade-two model of non-Newtonian fluids which for the first time allows nontrivial inflow boundary conditions. The new algorithm couples a Stokes equation for the fluid velocity with a transport equation for an auxiliary vector-valued function. As a third-order partial differential equation, the grade-two model requires an additional boundary condition, and our new formulation leads to a 
condition with a clear physical interpretation. We prove that the model is well posed using an iterative algorithm in function space by proving the iteration converges geometrically for sufficiently small data.

Finally, we demonstrated computationally that this algorithm can be successfully discretized. In subsequent work we will investigate the numerical discretization of the model in more detail.

\section{Appendix A. Spaces}

Here we collect the notation used for various Sobolev spaces and norms. We denote by $L^{p}(\Omega)$ the Lebesgue spaces [9] of $p$-th power integrable functions, with norm

$$
\|f\|_{L^{p}(\Omega)}=\left(\int_{\Omega}|f(\mathbf{x})|^{p} \mathrm{~d} x\right)^{1 / p} .
$$

Note that we can easily apply the same notation to vector or tensor valued $f$. We think of tensors of any arity as vectors of the appropriate length, and we think of $|f(\mathbf{x})|$ as the Euclidean length of this vector. For tensors of arity 2 (i.e., matrices) this is the same as the Frobenius norm. We will write the spaces for such tensor-valued functions as $L^{p}(\Omega)^{m}$ for the appropriate $m$ (e.g., $m=d^{2}$ for arity 2$)$. Similarly, we denote by $L^{\infty}(\Omega)$ the Lebesgue space of essentially bounded functions, with

$$
\|f\|_{L^{\infty}(\Omega)}=\sup \{|f(\mathbf{x})| \mid \text { a.e. } \mathbf{x} \in \Omega\} .
$$

Correspondingly, we define Sobolev spaces and norms of order $m$ by

$$
\|f\|_{W_{p}^{m}(\Omega)}=\left(\sum_{|\alpha| \leq m}\left\|D^{\alpha} f\right\|_{L^{p}(\Omega)}^{p}\right)^{1 / p},
$$

where $D^{\alpha}$ is the weak derivative $\partial^{\alpha} / \partial \mathbf{x}^{|\alpha|}[9]$. More precisely, the spaces $W_{p}^{m}(\Omega)$ are defined as the subspaces of $L^{p}(\Omega)$ for which the corresponding norm is finite. The case $p=2$ is denoted by $H$ :

$$
H^{m}(\Omega)=W_{2}^{m}(\Omega) .
$$

We will briefly use the space $H_{0}^{1}(\Omega)$ of $f \in H^{1}(\Omega)$ such that $f=0$ on $\partial \Omega$. The dual space $H^{-1}(\Omega)^{d}$ is the set of Schwartz distributions [25] for which the dual norm

$$
\|\mathbf{u}\|_{H^{-1}(\Omega)}=\sup _{\mathbf{0} \neq \boldsymbol{\phi} \in H_{0}^{1}(\Omega)^{d}} \frac{\langle\mathbf{u} \cdot \boldsymbol{\phi}\rangle}{\|\boldsymbol{\phi}\|_{H^{1}(\Omega)}}
$$

is finite.

\section{Appendix B. Determining inflow Boundary CONDitions}

The proposed method (3.9) requires specification of boundary conditions for $\mathbf{w}=-\Delta \mathbf{u}+\nabla \pi$. Here we compute the stress $\mathbf{w}$ for typical flow geometries.

\section{B.1. Grade-two channel flow}

To be specific, we define the domain $\Omega$ to be

$$
\Omega=\left\{\mathbf{x} \in \mathbb{R}^{2} \mid 0<x_{1}<L, 0<x_{2}<1\right\} .
$$

Suppose that $u_{2} \equiv 0$ and $u_{1}$ depends only on $x_{2}$. This is true for shear flow (Couette flow) and pressure-driven flow (Poiseuille flow). For the remainder of this subsection, we refer to $u_{1}$ as just $u$ to simplify notation. For such flows, $\mathbf{u} \cdot \nabla \mathbf{u}=\mathbf{0}$, and the strain rate $\nabla \mathbf{u}$ is given by

$$
\nabla \mathbf{u}=\left(\begin{array}{cc}
0 & u^{\prime} \\
0 & 0
\end{array}\right), \quad \nabla \mathbf{u}^{t}=\left(\begin{array}{cc}
0 & 0 \\
u^{\prime} & 0
\end{array}\right)
$$


Thus

$$
\begin{gathered}
\mathbf{A}=\left(\begin{array}{cc}
0 & u^{\prime} \\
u^{\prime} & 0
\end{array}\right), \quad \mathbf{u} \cdot \nabla \mathbf{A}=\mathbf{0}, \quad \mathbf{A} \circ \mathbf{A}=\left(\begin{array}{cc}
\left(u^{\prime}\right)^{2} & 0 \\
0 & \left(u^{\prime}\right)^{2}
\end{array}\right)=\left(u^{\prime}\right)^{2} \mathcal{I}, \\
\mathbf{A} \circ(\nabla \mathbf{u})=\left(\begin{array}{cc}
0 & 0 \\
0 & \left(u^{\prime}\right)^{2}
\end{array}\right), \quad(\nabla \mathbf{u})^{t} \circ \mathbf{A}=\left(\mathbf{A}^{t} \circ(\nabla \mathbf{u})\right)^{t}=(\mathbf{A} \circ(\nabla \mathbf{u}))^{t}=\left(\begin{array}{cc}
0 & 0 \\
0 & \left(u^{\prime}\right)^{2}
\end{array}\right), \\
(\nabla \mathbf{u}) \circ \mathbf{A}=\left(\begin{array}{cc}
\left(u^{\prime}\right)^{2} & 0 \\
0 & 0
\end{array}\right), \quad \mathbf{A} \circ(\nabla \mathbf{u})^{t}=\left((\nabla \mathbf{u}) \circ \mathbf{A}^{t}\right)^{t}=((\nabla \mathbf{u}) \circ \mathbf{A})^{t}=\left(\begin{array}{cc}
\left(u^{\prime}\right)^{2} & 0 \\
0 & 0
\end{array}\right) .
\end{gathered}
$$

We can use the formula (2.2) to compute the stress:

$$
\begin{aligned}
\mathbf{T}_{\mathrm{G}} & =\nu\left(\begin{array}{cc}
0 & u^{\prime} \\
u^{\prime} & 0
\end{array}\right)+2 \alpha_{1}\left(\begin{array}{cc}
0 & 0 \\
0 & \left(u^{\prime}\right)^{2}
\end{array}\right)+\alpha_{2}\left(\begin{array}{cc}
\left(u^{\prime}\right)^{2} & 0 \\
0 & \left(u^{\prime}\right)^{2}
\end{array}\right) \\
& =\mathbf{T}_{\mathrm{N}}+\left(u^{\prime}\right)^{2}\left(\begin{array}{cc}
\alpha_{2} & 0 \\
0 & 2 \alpha_{1}+\alpha_{2}
\end{array}\right) .
\end{aligned}
$$

The tensor $\boldsymbol{\tau}$ is given by (2.10):

$$
\begin{aligned}
\boldsymbol{\tau} & =\alpha_{1}(\nabla \mathbf{u})^{t} \circ \mathbf{A}+\left(\alpha_{1}+\alpha_{2}\right) \mathbf{A} \circ \mathbf{A}-\mathbf{u} \otimes \mathbf{u} \\
& =\alpha_{1}\left(\begin{array}{cc}
0 & 0 \\
0 & \left(u^{\prime}\right)^{2}
\end{array}\right)+\left(\alpha_{1}+\alpha_{2}\right)\left(\begin{array}{cc}
\left(u^{\prime}\right)^{2} & 0 \\
0 & \left(u^{\prime}\right)^{2}
\end{array}\right)-\left(\begin{array}{cc}
u^{2} & 0 \\
0 & 0
\end{array}\right) \\
& =\left(u^{\prime}\right)^{2}\left(\begin{array}{cc}
\alpha_{1}+\alpha_{2} & 0 \\
0 & 2 \alpha_{1}+\alpha_{2}
\end{array}\right)-\left(\begin{array}{cc}
u^{2} & 0 \\
0 & 0
\end{array}\right) .
\end{aligned}
$$

We can compute $\nabla \cdot \boldsymbol{\tau}$ as follows. By definition, $(\nabla \cdot \boldsymbol{\tau})_{i}=\sum_{j} \tau_{i j, j}=\tau_{i 2,2}$ since $\boldsymbol{\tau}$ is constant in $x_{1}$ and thus $\tau_{i 1,1}=0$. Therefore

$$
\nabla \cdot \boldsymbol{\tau}=\left(\begin{array}{c}
\tau_{12,2} \\
\tau_{22,2}
\end{array}\right)=\left(\begin{array}{c}
0 \\
\tau_{22,2}
\end{array}\right)=\left(2 \alpha_{1}+\alpha_{2}\right) 2 u^{\prime} u^{\prime \prime}\left(\begin{array}{l}
0 \\
1
\end{array}\right)
$$

Similarly,

$$
N(\mathbf{u}, \pi)=-\alpha_{1} \pi \nabla \mathbf{u}^{t}+\boldsymbol{\tau}=-\alpha_{1} \pi\left(\begin{array}{cc}
0 & 0 \\
u^{\prime} & 0
\end{array}\right)+\left(u^{\prime}\right)^{2}\left(\begin{array}{cc}
\alpha_{1}+\alpha_{2} & 0 \\
0 & 2 \alpha_{1}+\alpha_{2}
\end{array}\right)-\left(\begin{array}{cc}
u^{2} & 0 \\
0 & 0
\end{array}\right),
$$

and from (3.5) we find

$$
\nabla \cdot N(\mathbf{u}, \pi)=-\alpha_{1} u^{\prime}\left(\begin{array}{c}
0 \\
\pi_{x_{1}}
\end{array}\right)+\left(2 \alpha_{1}+\alpha_{2}\right) 2 u^{\prime} u^{\prime \prime}\left(\begin{array}{l}
0 \\
1
\end{array}\right) .
$$

For shear flow, $u$ is linear, so $u^{\prime}$ is constant, and thus $\mathbf{T}_{\mathrm{G}}$ is constant. Therefore $\nabla \cdot \mathbf{T}_{\mathrm{G}} \equiv \mathbf{0}$. Similarly, $\Delta \mathbf{u} \equiv \mathbf{0}$, and $p$ is constant. Suppose that $p_{0}$ is this constant. If we specify that $\left.\pi\right|_{\Gamma_{-}}=p_{0}$, then we conclude that $\pi$ is also constant $\left(\pi=p_{0}\right)$. Thus $\mathbf{w}=\mathbf{0}$ as well. But there could be other solutions for other choices of $\left.\pi\right|_{\Gamma_{-}}$, leading to nonconstant $\pi$. In that case, $\mathbf{w}=\nabla \pi \neq \mathbf{0}$.

\section{B.2. Poiseuille flow}

For Poiseuille flow, $u$ is quadratic, and $\nabla \cdot \mathbf{T}_{\mathrm{G}}$ is not even constant. Since $\mathbf{u} \cdot \nabla \mathbf{u}=\mathbf{0}$, the top equation in (2.3) takes the form

$$
\left(\begin{array}{c}
-\nu u^{\prime \prime}+p_{x_{1}} \\
p_{x_{2}}
\end{array}\right)=-\nu \Delta \mathbf{u}+\nabla p=\nabla \cdot\left(\mathbf{T}_{\mathrm{G}}-\mathbf{T}_{\mathrm{N}}\right)=\left(\begin{array}{c}
0 \\
\left(2 \alpha_{1}+\alpha_{2}\right)\left(\left(u^{\prime}\right)^{2}\right)^{\prime}
\end{array}\right) .
$$

Then we get two equations for the pressure:

$$
p_{x_{1}}=\nu u^{\prime \prime}, \quad p_{x_{2}}=\left(2 \alpha_{1}+\alpha_{2}\right)\left(\left(u^{\prime}\right)^{2}\right)^{\prime} .
$$


Define

$$
p(\mathbf{x})=\nu u^{\prime \prime} x_{1}+\left(2 \alpha_{1}+\alpha_{2}\right)\left(u^{\prime}\right)^{2}+c_{p} .
$$

This function satisfies the required equations for the pressure for any constant $c_{p}$.

The equation relating $p$ and $\pi$ is $p=\nu \pi+\alpha_{1} u \pi_{x_{1}}$, so

$$
\nu \pi+\alpha_{1} u \pi_{x_{1}}=\nu u^{\prime \prime} x_{1}+\left(2 \alpha_{1}+\alpha_{2}\right)\left(u^{\prime}\right)^{2}+c_{p} .
$$

Let us make the ansatz that $\pi(\mathbf{x})=u^{\prime \prime} x_{1}+f\left(x_{2}\right)$. Computing, we find

$$
\begin{aligned}
\nu \pi+\alpha_{1} u \pi_{x_{1}} & =\nu u^{\prime \prime} x_{1}+\nu f\left(x_{2}\right)+\alpha_{1} u u^{\prime \prime} \\
& =p(\mathbf{x})-\left(2 \alpha_{1}+\alpha_{2}\right)\left(u^{\prime}\right)^{2}-c_{p}+\nu f\left(x_{2}\right)+\alpha_{1} u u^{\prime \prime} .
\end{aligned}
$$

Thus our ansatz if valid if

$$
f\left(x_{2}\right)=\nu^{-1}\left(\left(2 \alpha_{1}+\alpha_{2}\right)\left(u^{\prime}\right)^{2}+c_{p}-\alpha_{1} u u^{\prime \prime}\right) .
$$

Therefore

$$
\pi(\mathbf{x})=u^{\prime \prime} x_{1}+\nu^{-1}\left(\left(2 \alpha_{1}+\alpha_{2}\right)\left(u^{\prime}\right)^{2}-\alpha_{1} u u^{\prime \prime}\right)+\frac{c_{p}}{\nu} .
$$

Applying (B.8) to (B.4), we get

$$
\nabla \cdot N(\mathbf{u}, \pi)=-\alpha_{1} u^{\prime} u^{\prime \prime}\left(\begin{array}{l}
0 \\
1
\end{array}\right)+\left(2 \alpha_{1}+\alpha_{2}\right) 2 u^{\prime} u^{\prime \prime}\left(\begin{array}{l}
0 \\
1
\end{array}\right)=\left(3 \alpha_{1}+2 \alpha_{2}\right) u^{\prime} u^{\prime \prime}\left(\begin{array}{l}
0 \\
1
\end{array}\right) .
$$

Let us check the first equation in (3.8). We have (recall that $u^{\prime \prime \prime}=0$ )

$$
\Delta \mathbf{u}=u^{\prime \prime}\left(\begin{array}{l}
1 \\
0
\end{array}\right), \quad \nabla \pi=\left(\begin{array}{c}
u^{\prime \prime} \\
\nu^{-1}\left(3 \alpha_{1}+2 \alpha_{2}\right) u^{\prime} u^{\prime \prime}
\end{array}\right) \quad \Longrightarrow \quad \mathbf{w}=\left(\begin{array}{c}
0 \\
\nu^{-1}\left(3 \alpha_{1}+2 \alpha_{2}\right) u^{\prime} u^{\prime \prime}
\end{array}\right) .
$$

Note that $\mathbf{w}_{, x_{1}} \equiv 0$. Thus (3.8) implies that

$$
\nabla \cdot N=\nu \mathbf{w}
$$

which is consistent with (B.9). Thus (B.10) gives a boundary condition for the inflow boundary $\Gamma_{-}$suitable for use in the algorithm (3.9) to compute Poiseuille flow. More importantly, it can be used for more complex pressure-driven flows in which the inlet is a two-dimensional channel.

To summarize, for shear (Couette) flow, $u^{\prime \prime}=0$, so $\mathbf{w}=\mathbf{0}$. For Poiseuille flow, in the channel (B.1),

$$
u=U x_{2}\left(L-x_{2}\right), \quad u^{\prime}=U\left(L-2 x_{2}\right), \quad u^{\prime \prime}=-2 U, \quad u^{\prime} u^{\prime \prime}=2 U^{2}\left(2 x_{2}-L\right),
$$

so we can take

$$
\mathbf{g}=\mathbf{u}=U\left(\begin{array}{c}
x_{2}\left(L-x_{2}\right) \\
0
\end{array}\right), \quad \mathbf{w}=-\frac{2 U^{2}}{\nu}\left(L-2 x_{2}\right)\left(\begin{array}{c}
0 \\
2 \alpha_{2}+3 \alpha_{1}
\end{array}\right)
$$

Acknowledgements. The authors would like to thank the anonymous referees for their useful suggestions which provided further clarity to this paper. SP is supported in part by the National Science Foundation NSF DMS-2011519.

\section{REFERENCES}

[1] N. Arada, P. Correia and A. Sequeira, Analysis and finite element simulations of a second-order fluid model in a bounded domain. Numer. Methods Part. Differ. Equ. Int. J. 23 (2007) 1468-1500.

[2] H.A. Barnes, J.F. Hutton and K. Walters, An Introduction to Rheology. Vol. 3. Elsevier (1989).

[3] J.-M. Bernard, Solutions globales variationnelles et classiques des fluides de grade deux. C. R. Acad. Sci. Ser. I Math. 327 (1998) 953-958. 
[4] J.-M. Bernard, Stationary problem of second-grade fluids in three dimensions: existence, uniqueness and regularity. Math. Methods Appl. Sci. 22 (1999) 655-687.

[5] J.-M. Bernard, Problem of second grade fluids in convex polyhedrons. SIAM J. Math. Anal. 44 (2012) $2018-2038$.

[6] J.-M. Bernard, Steady transport equation in the case where the normal component of the velocity does not vanish on the boundary. SIAM J. Math. Anal. 44 (2012) 993-1018.

[7] J.-M. Bernard, Solutions in $H^{1}$ of the steady transport equation in a bounded polygon with a full non-homogeneous velocity. J. Math. Pures App. 107 (2017) 697-736.

[8] J.-M. Bernard, Fully nonhomogeneous problem of two-dimensional second grade fluids. Math. Methods Appl. Sci. 41 (2018) $6772-6792$.

[9] S.C. Brenner and L.R. Scott, The Mathematical Theory of Finite Element Methods, 3rd edition. Springer-Verlag (2008).

[10] D. Cioranescu, V. Girault and K.R. Rajagopal, Mechanics and Mathematics of Fluids of the Differential Type. In Vol. 35 of Advances in Mechanics and Mathematics. Springer (2016).

[11] J.L. Ericksen and R.S. Rivlin, Stress-deformation relations for isotropic materials. Arch. Ration. Mech. Anal. 4 (1955) $323-425$.

[12] B.A. Gecim, Non-Newtonian effects of multigrade oils on journal bearing performance. Tribol. Trans. 33 (1990) $384-394$.

[13] V. Girault and P.-A. Raviart, Finite Element Methods for Navier-Stokes Equations. Springer Verlag, Berlin (1986).

[14] V. Girault and L.R. Scott, Finite element discretizations of a two-dimensional grade-two fluid model. ESAIM: M2AN 35 (2001) $1007-1053$.

[15] V. Girault and L.R. Scott, Analysis of a two-dimensional grade-two fluid model with a tangential boundary condition. J. Math. Pures Appl. 78 (1999) 981-1011.

[16] V. Girault and L.R. Scott, Wellposedness of some Oldroyd models that lack explicit dissipation. Research Report UC/CS TR-2017-04, Dept. Comp. Sci., Univ. Chicago (2017).

[17] D. Gómez-Díaz and J.M. Navaza, Rheology of aqueous solutions of food additives: effect of concentration, temperature and blending. J. Food Eng. 56 (2003) 387-392.

[18] L.D. Landau and E.M. Lifshitz, Fluid Mechanics. Pergamon Press (1959).

[19] R. Lapasin, Rheology of Industrial Polysaccharides: Theory and Applications. Springer Science \& Business Media (2012).

[20] A.S. Lodge, Low-shear-rate rheometry and polymer quality control. Chem. Eng. Commun. 32 (1985) 1-60.

[21] A.S. Lodge, W.G. Pritchard and L.R. Scott, The hole-pressure problem. IMA J. Appl. Math. 46 (1991) 39-66.

[22] H. Morgan and L. Ridgway Scott, Towards a unified finite element method for the Stokes equations. SIAM J. Sci. Comput. 40 (2018) A130-A141.

[23] M. Nyström, H.R. Tamaddon Jahromi, M. Stading and M.F. Webster, Hyperbolic contraction measuring systems for extensional flow. Mech. Time-Depend. Mater. 21 (2017) 455-479.

[24] S. Pollock and L. Ridgway Scott, Transport equations with inflow boundary conditions. Submitted (2022).

[25] L. Schwartz, Théorie des Distributions. Hermann, Paris (1966).

[26] L.R. Scott, $C^{1}$ piecewise polynomials satisfying boundary conditions. Research Report UC/CS TR-2019-18, Dept. Comp. Sci., Univ. Chicago (2019).

[27] T.W. Selby, The non-Newtonian characteristics of lubricating oils. ASLE Trans. 1 (1958) 68-81.

[28] P.A. Vasquez, Y. Jin, E. Palmer, D. Hill and M. Gregory Forest, Modeling and simulation of mucus flow in human bronchial epithelial cell cultures - Part I: idealized axisymmetric swirling flow. PLoS Comput. Biol. 12 (2016) 1-28.

\section{Subscribe to Open (S20) A fair and sustainable open access model}

This journal is currently published in open access under a Subscribe-to-Open model (S2O). S2O is a transformative model that aims to move subscription journals to open access. Open access is the free, immediate, online availability of research articles combined with the rights to use these articles fully in the digital environment. We are thankful to our subscribers and sponsors for making it possible to publish this journal in open access, free of charge for authors.

\section{Please help to maintain this journal in open access!}

Check that your library subscribes to the journal, or make a personal donation to the S2O programme, by contacting subscribers@edpsciences.org

More information, including a list of sponsors and a financial transparency report, available at: https://www. edpsciences.org/en/maths-s2o-programme 